CRYSTALLOGRAPHIC COMMUNICATIONS

ISSN 2056-9890

Received 15 June 2021

Accepted 23 August 2021

Edited by G. Diaz de Delgado, Universidad de Los Andes, Venezuela

Keywords: crystal structure; 4-aminoquinazoline; 6-aminoquinazolin-4(3H)-one; 6 nitroquinazolin-4(3H)-one; hemihydrochloride.

CCDC references: $2104939 ; 2104938$; 2104937

Supporting information: this article has supporting information at journals.iucr.org/e

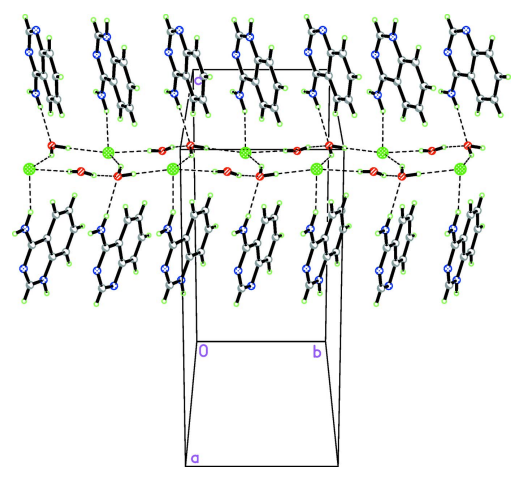

OPEN ӘACCESS

\section{Crystal structures of 6-nitroquinazolin-4(3H)-one, 6-aminoquinazolin-4(3H)-one and 4-amino- quinazoline hemihydrochloride dihydrate}

\author{
Kambarali Turgunov, ${ }^{\mathrm{a}, \mathrm{b} *}$ Mirjalol Ziyadullaev, ${ }^{\mathrm{a}}$ Farkhod Khoshimov, ${ }^{\mathrm{c}}$ Rikhsiboy \\ Karimov $^{\mathrm{a}}$ and Burkhon Elmuradov ${ }^{\mathrm{a}}$
}

\begin{abstract}
${ }^{a}$ S. Yunusov Institute of the Chemistry of Plant Substances, Academy of Sciences of Uzbekistan, Mirzo Ulugbek Str., 77, Tashkent 100170, Uzbekistan, ' Turin Polytechnic University in Tashkent, Kichik Khalka Yuli Str. 17, Tashkent 100095, Uzbekistan, and ${ }^{\mathbf{C}}$ Namangan Institute of Engineering and Technology, Kosonsoy Str., 7, Namangan 160115, Uzbekistan. *Correspondence e-mail: kk_turgunov@rambler.ru
\end{abstract}

The title compounds, 6-nitroquinazolin- $4(3 H)$-one $\left(\mathrm{C}_{8} \mathrm{H}_{5} \mathrm{~N}_{3} \mathrm{O}_{3} ;\right.$ I $)$, 6-aminoquinazolin-4(3H)-one $\left(\mathrm{C}_{8} \mathrm{H}_{7} \mathrm{~N}_{3} \mathrm{O} ; \mathbf{I I}\right)$ and 4-aminoquinazolin-1-ium chloride-4aminoquinazoline-water $(1 / 1 / 2),\left(\mathrm{C}_{8} \mathrm{H}_{8} \mathrm{~N}_{3}^{+} \cdot \mathrm{Cl}^{-} \cdot \mathrm{C}_{8} \mathrm{H}_{7} \mathrm{~N}_{3} \cdot 2 \mathrm{H}_{2} \mathrm{O}\right.$; III) were synthesized and their structures were determined by single-crystal $\mathrm{X}$-ray analysis. In the crystals of $\mathbf{I}$ and II, the quinazoline molecules form hydrogen-bonded dimers via $\mathrm{N}-\mathrm{H} \cdots \mathrm{O}$ interactions. The dimers are connected by weak intermolecular $\mathrm{C}-\mathrm{H} \cdots \mathrm{N}$ and $\mathrm{C}-\mathrm{H} \cdots \mathrm{O}$ hydrogen bonds, forming a layered structure in the case of $\mathbf{I}$. In the crystal of II, N-H $\cdots \mathrm{N}$ and $\mathrm{C}-\mathrm{H} \cdots \mathrm{O}$ interactions link the dimers into a three-dimensional network structure. The asymmetric unit of III consists of two quinazoline molecules, one of which is protonated, a chloride ion, and two water molecules. The chloride anion and the water molecules form hydrogen-bonded chains consisting of fused five-membered rings. The protonated and unprotonated quinazolin molecules are linked to the chloride ions and water molecules of the chain by their amino groups.

\section{Chemical context}

Heterocyclic compounds play an important role in the lives of plant and living organisms because of their properties, including anti-inflammatory (Azab et al., 2016), antitumor (Ishikawa et al., 2009), antiviral (De Clercq \& Field, 2006) and other activities (Ding et al., 1999). Quinazoline derivatives occupy a distinct position among nitrogen-containing heterocycles because of their wide spectrum of pharmaceutical and biopharmaceutical properties, amongst them anticancer (Chandregowda et al., 2009), antibacterial (Antipenko et al., 2009), anti-inflammatory (Alagarsamy et al., 2007), antituberculosis (Nandy et al., 2006), antihypertension (Hess et al., 1968) and antidiabetic (Paneersalvam et al., 2010) activities.
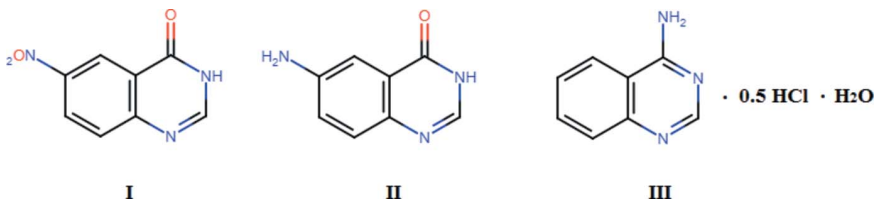

In line with this, we synthesized 6-nitroquinazolin-4(3H)one (I), 6-aminoquinazolin-4(3H)-one (II) and 4-aminoquinazoline hemihydrochloride dihydrate (III), which are important intermediates in drug synthesis, and their crystal structures were determined. The hemi-protonated structures may be useful for the preparation of materials important to 
Table 1

Selected bond lengths $(\AA)$ for $\mathbf{I}$.

\begin{tabular}{llll}
\hline $\mathrm{N} 1-\mathrm{C} 2$ & $1.287(3)$ & $\mathrm{N} 3-\mathrm{C} 4$ & $1.366(3)$ \\
$\mathrm{C} 2-\mathrm{N} 3$ & $1.354(3)$ & $\mathrm{C} 6-\mathrm{N} 9$ & $1.464(3)$ \\
\hline
\end{tabular}

Table 2

Selected bond lengths $(\AA)$ for II.

\begin{tabular}{llll}
\hline $\mathrm{N} 1 A-\mathrm{C} 2 A$ & $1.291(5)$ & $\mathrm{N} 1 B-\mathrm{C} 2 B$ & $1.290(5)$ \\
$\mathrm{C} 2 A-\mathrm{N} 3 A$ & $1.369(4)$ & $\mathrm{C} 2 B-\mathrm{N} 3 B$ & $1.364(4)$ \\
$\mathrm{N} 3 A-\mathrm{C} 4 A$ & $1.376(4)$ & $\mathrm{N} 3 B-\mathrm{C} 4 B$ & $1.366(4)$ \\
$\mathrm{C} 6 A-\mathrm{N} 9 A$ & $1.374(4)$ & $\mathrm{C} 6 B-\mathrm{N} 9 B$ & $1.392(5)$ \\
\hline
\end{tabular}

Table 3

Selected bond lengths $(\AA)$ for III.

\begin{tabular}{llll}
\hline $\mathrm{N} 1 A-\mathrm{C} 2 A$ & $1.315(4)$ & $\mathrm{N} 1 B-\mathrm{C} 2 B$ & $1.309(4)$ \\
$\mathrm{C} 2 A-\mathrm{N} 3 A$ & $1.328(4)$ & $\mathrm{C} 2 B-\mathrm{N} 3 B$ & $1.340(4)$ \\
$\mathrm{N} 3 A-\mathrm{C} 4 A$ & $1.363(4)$ & $\mathrm{N} 3 B-\mathrm{C} 4 B$ & $1.347(4)$ \\
$\mathrm{C} 4 A-\mathrm{N} 9 A$ & $1.293(4)$ & $\mathrm{C} 4 B-\mathrm{N} 9 B$ & $1.323(4)$ \\
\hline
\end{tabular}

various branches of science, ranging from biology to nanodevice fabrication and to pharmaceuticals (Perumalla et al., 2013).

\section{Structural commentary}

Compound $\mathbf{I}$ crystallizes in the triclinic space group $P \overline{1}$ with one molecule in the asymmetric unit. As a whole, the molecule is nearly planar. The nitro group is rotated slightly with respect to the quinazoline ring system, the $\mathrm{C} 5-\mathrm{C} 6-\mathrm{N} 9-\mathrm{O} 3$ and $\mathrm{C} 7-\mathrm{C} 6-\mathrm{N} 9-\mathrm{O} 2$ torsion angles being $6.0(3)$ and $4.9(4)^{\circ}$, respectively. All bond lengths and angles are normal and in good agreement with those reported previously (Liao et al., 2018; Yong et al., 2008). Fig. 1 shows the molecular structure of I in the solid state. Selected geometric parameters are listed in Table 1.

Compound II crystallizes in the orthorhombic space group $P c a 2_{1}$ with two crystallographically independent molecules, $A$

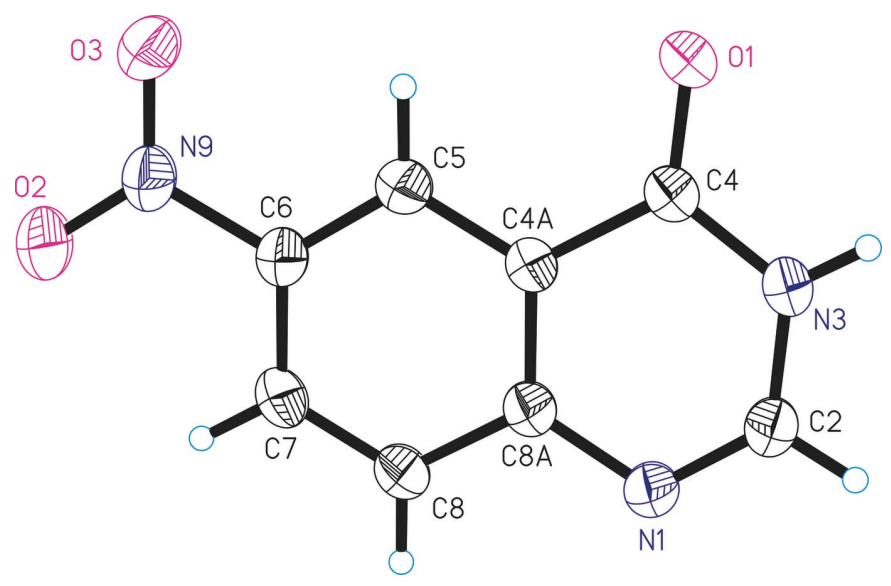

Figure 1

The molecular structure of 6-nitroquinazolin-4(3H)-one (I), with displacement ellipsoids drawn at the $50 \%$ probability level.

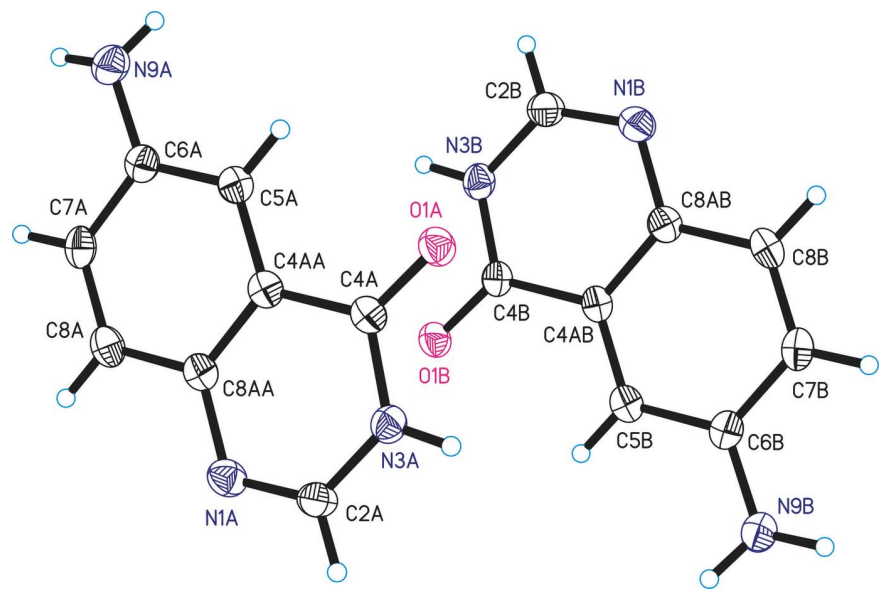

Figure 2

The molecular structure of 6-aminoquinazolin-4(3H)-one (II), showing the two independent molecules, with displacement ellipsoids drawn at the $50 \%$ probability level.

and $B$, in the asymmetric unit (Fig. 2). All the atoms of the molecule (except the amino-group hydrogens) lie in the same plane. The nitrogen atom of the amino group is somewhere between the $s p^{2}$ and $s p^{3}$ hybridized states, the sum of the valence angles at the nitrogen atom being 349 and $342^{\circ}$ in molecules $A$ and $B$, respectively. All bond lengths and angles

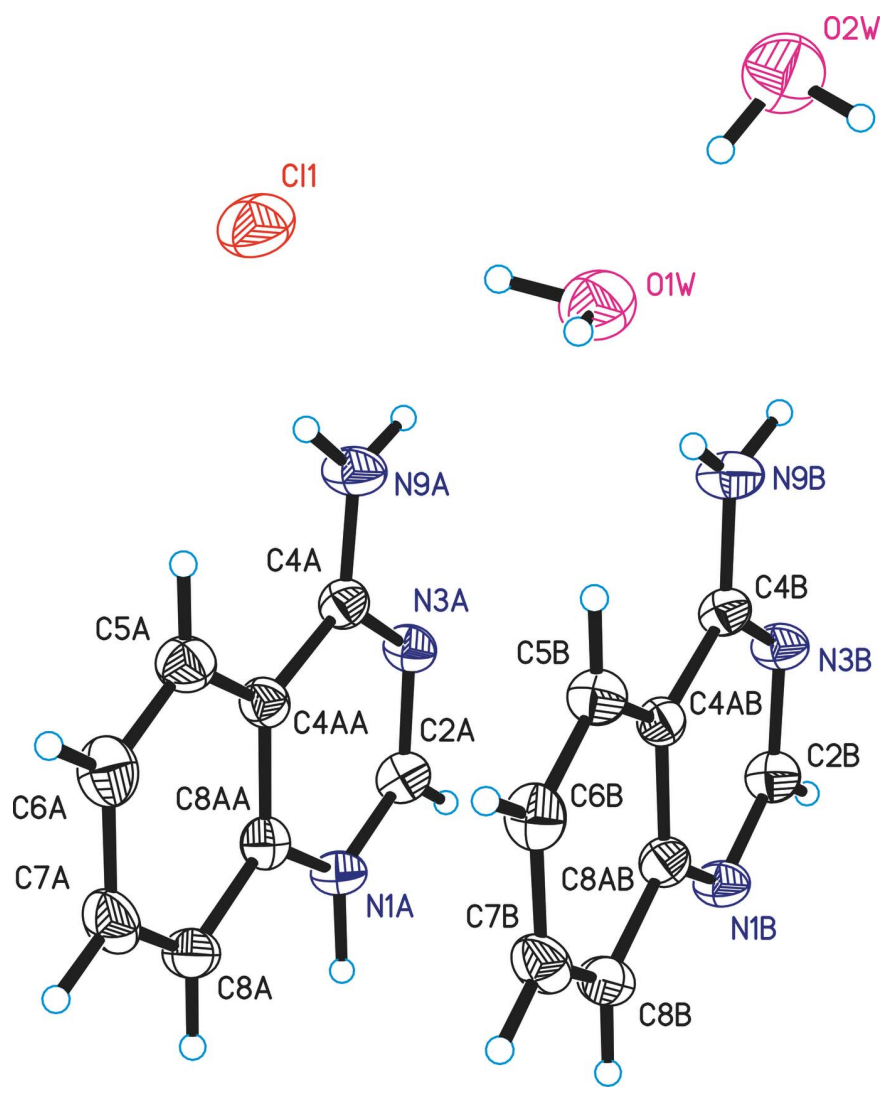

Figure 3

The asymmetric unit of compound III with displacement ellipsoids drawn at the $50 \%$ probability level. 
Table 4

Hydrogen-bond geometry $\left(\AA,^{\circ}\right)$ for $\mathbf{I}$.

\begin{tabular}{lllll}
\hline$D-\mathrm{H} \cdots A$ & $D-\mathrm{H}$ & $\mathrm{H} \cdots A$ & $D \cdots A$ & $D-\mathrm{H} \cdots A$ \\
\hline $\mathrm{N} 3-\mathrm{H} 3 \cdots \mathrm{O} 1^{\mathrm{i}}$ & $0.80(3)$ & $2.02(3)$ & $2.814(2)$ & $178(4)$ \\
$\mathrm{C} 8-\mathrm{H} 8 \cdots \mathrm{N} 1^{\mathrm{ii}}$ & 0.93 & 2.53 & $3.450(3)$ & 172 \\
$\mathrm{C} 2-\mathrm{H} 2 \cdots 2^{\mathrm{iii}}$ & 0.93 & 2.57 & $3.466(4)$ & 163 \\
$\mathrm{C} 7-\mathrm{H} 7 \cdots \mathrm{O} 2^{\text {iv }}$ & 0.93 & 2.56 & $3.437(3)$ & 158 \\
\hline
\end{tabular}

Symmetry codes: (i) $-x+1,-y,-z+1$; (ii) $-x,-y+1,-z+2$; (iii) $x-1, y-1, z$; (iv) $-x+1,-y+2,-z+2$.

are normal. Selected geometric parameters are listed in Table 2.

In the case of compound III, there are protonated $(A)$ and unprotonated $(B)$ 4-aminoquinazoline molecules (Fig. 3) in the asymmetric unit and they both have a planar structure. Molecule $A$ is protonated at the N1 nitrogen atom and this leads to an elongation of the $\mathrm{N} 1-\mathrm{C} 2$ and N3-C4 bonds and a shortening of the $\mathrm{C} 2-\mathrm{N} 3$ and $\mathrm{C} 4-\mathrm{N} 9$ bonds with respect to the unprotonated molecule $B$. In both $A$ and $B$, the nitrogen atom of the amino group is in an $s p^{2}$ hybridized state. The sum of the valence angles around the nitrogen atoms in molecules $A$ and $B$ are 360 and $359^{\circ}$, respectively, and the carbon-toamino group nitrogen bond lengths $\mathrm{C} 4-\mathrm{N} 9$ are shorter than the bond lengths observed in compound II (Table 3).

\section{Supramolecular features}

In the crystal of $\mathbf{I}$, intermolecular $\mathrm{N}-\mathrm{H} \cdots \mathrm{O}$ hydrogen bonds link the molecules into centrosymmetric dimers, forming $R_{2}^{2}(8)$ motifs. Other head-to-head $R_{2}^{2}(10)$ and $R_{2}^{2}(8)$ motifs are formed by weak intermolecular $\mathrm{C}-\mathrm{H} \cdots \mathrm{O}$ and $\mathrm{C}-\mathrm{H} \cdots \mathrm{N}$ hydrogen bonds, producing layers parallel to the (112) plane (Table 4, Fig. 4). In addition, an $R_{3}^{2}(8)$ ring motif is formed by the interactions between three adjacent molecules. The layers are linked though $\pi-\pi$ stacking interactions with centroidcentroid distances of 3.8264 (13) and 3.9600 (14) $\AA$ into a three-dimensional network.

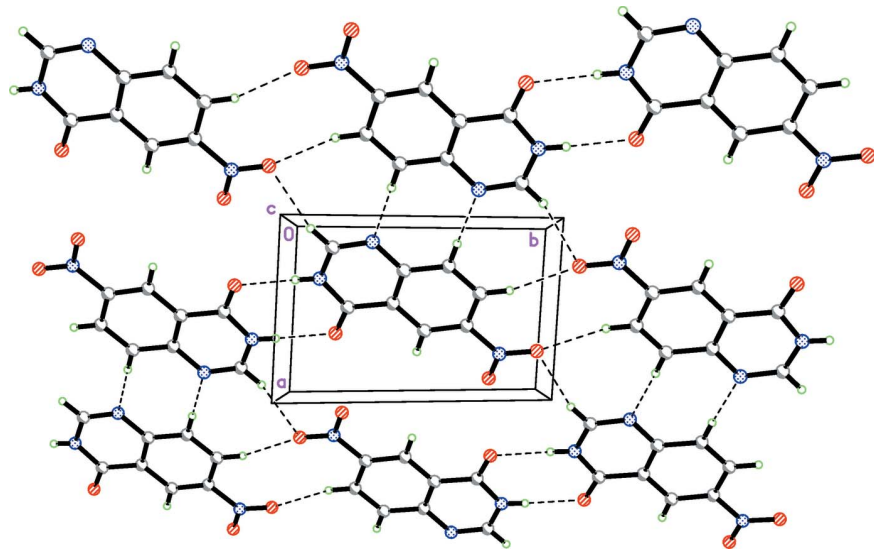

Figure 4

Hydrogen bonding in the crystal of 6-nitroquinazolin-4(3H)-one (I). Colour code: $\mathrm{C}$ grey, $\mathrm{H}$ green, $\mathrm{N}$ blue, $\mathrm{O}$ red.
Table 5

Hydrogen-bond geometry $\left(\AA{ }^{\circ}\right)$ for II.

\begin{tabular}{lclll}
\hline$D-\mathrm{H} \cdots A$ & $D-\mathrm{H}$ & $\mathrm{H} \cdots A$ & $D \cdots A$ & $D-\mathrm{H} \cdots A$ \\
\hline $\mathrm{N} 9 A-\mathrm{H} 9 A A \cdots \mathrm{N} 9 B^{\mathrm{i}}$ & $0.93(4)$ & $2.55(4)$ & $3.435(5)$ & $160(4)$ \\
$\mathrm{N} 9 A-\mathrm{H} 9 A B \cdots \mathrm{N} 1 A^{\text {ii }}$ & $0.81(4)$ & $2.34(4)$ & $3.144(5)$ & $170(4)$ \\
$\mathrm{N} 9 B-\mathrm{H} 9 B B \cdots \mathrm{N} 1 B^{\text {iii }}$ & $0.91(4)$ & $2.19(4)$ & $3.092(5)$ & $174(4)$ \\
$\mathrm{N} 3 A-\mathrm{H} 3 A \cdots \mathrm{O} 1 B^{\text {iv }}$ & $0.95(3)$ & $1.89(3)$ & $2.832(4)$ & $175(3)$ \\
$\mathrm{N} 3 B-\mathrm{H} 3 B \cdots \mathrm{O} 1 A^{\mathrm{v}}$ & $0.92(4)$ & $1.93(4)$ & $2.847(3)$ & $173(4)$ \\
\hline
\end{tabular}

Symmetry codes: (i) $-x+1,-y+1, z-\frac{1}{2}$; (ii) $x-\frac{1}{2},-y+1, z$; (iii) $x+\frac{1}{2},-y, z$; (iv) $x, y-1, z ;(\mathrm{v}) x, y+1, z$.

The two independent molecules of compound II are related by a pseudo-center of symmetry and are linked by two $\mathrm{N}-$ $\mathrm{H}$... O hydrogen bonds, forming an $R_{2}^{2}(8)$ motif. An $\mathrm{N}-$ $\mathrm{H} \cdots \mathrm{N}$ hydrogen bond generates a three-dimensional network (Table 5, Fig. 5).

The packing analysis of III shows that the protonated and unprotonated 4-aminoquinazoline molecules are linked by intermolecular $\mathrm{N}-\mathrm{H} \cdots \mathrm{N}$ hydrogen bonds, forming pseudocentrosymmetric dimers characterized by a donor-acceptor distance of 2.786 (3) $\AA$. Other $\mathrm{N}-\mathrm{H} \cdots \mathrm{N}$ hydrogen bonds form centrosymmetric $R_{2}^{2}(8)$ ring motifs. The chloride anion and water molecules form hydrogen-bonded chains consisting of fused five-membered rings with the participation of two chloride anions and three water molecules. A chain of rings runs through the twofold screw axis parallel to the [010] direction (Fig. 6). The protonated and unprotonated quinazoline molecules link to the chain via $\mathrm{N}-\mathrm{H} \cdots \mathrm{Cl}$ and $\mathrm{N}-$ $\mathrm{H}$... Ow hydrogen bonds from the lower and upper side (Table 6, Fig. 6). The chain direction corresponds to the smallest unit-cell edge and such self-assembly of molecules has also been observed in other quinazoline hydrochloride crystals

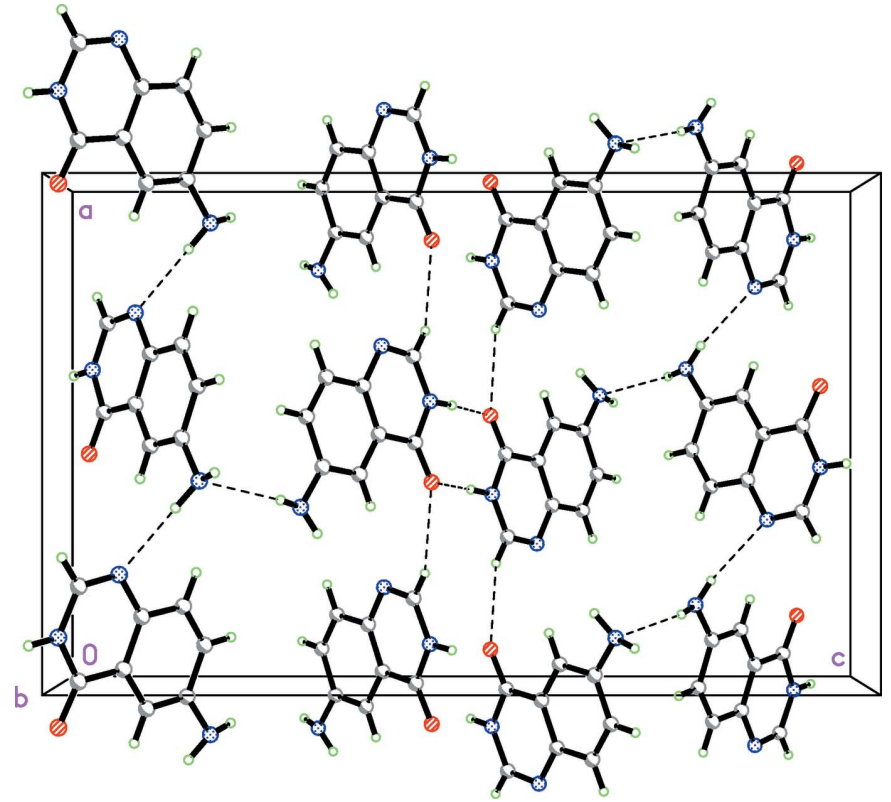

Figure 5

Hydrogen bonding in the crystal of 6-aminoquinazolin-4(3H)-one (II). Colour code: $\mathrm{C}$ grey, $\mathrm{H}$ green, $\mathrm{N}$ blue, $\mathrm{O}$ red. 
Table 6

Hydrogen-bond geometry $\left(\AA{ }^{\circ}\right)$ for III.

\begin{tabular}{lllll}
\hline$D-\mathrm{H} \cdots A$ & $D-\mathrm{H}$ & $\mathrm{H} \cdots A$ & $D \cdots A$ & $D-\mathrm{H} \cdots A$ \\
\hline $\mathrm{N} 1 A-\mathrm{H} 1 A \cdots \mathrm{N} 1 B^{\mathrm{i}}$ & $1.03(3)$ & $1.76(3)$ & $2.786(3)$ & $173(3)$ \\
$\mathrm{N} 9 A-\mathrm{H} 9 A A \cdots \mathrm{N} 3 B^{\mathrm{ii}}$ & $0.91(4)$ & $2.00(4)$ & $2.907(4)$ & $175(3)$ \\
$\mathrm{N} 9 A-\mathrm{H} 9 A B \cdots \mathrm{Cl} 1$ & $0.94(5)$ & $2.34(5)$ & $3.206(2)$ & $153(5)$ \\
$\mathrm{N} 9 B-\mathrm{H} 9 B A \cdots \mathrm{N} 3 A^{\mathrm{ii}}$ & $0.96(4)$ & $2.12(4)$ & $3.074(4)$ & $174(3)$ \\
$\mathrm{N} 9 B-\mathrm{H} 9 B B \cdots \mathrm{O} 1 W$ & $0.79(4)$ & $2.22(3)$ & $2.999(4)$ & $167(4)$ \\
$\mathrm{O} 1 W-\mathrm{H} 1 W 1 \cdots \mathrm{Cl} 1$ & $0.90(3)$ & $2.25(3)$ & $3.157(4)$ & $178(6)$ \\
$\mathrm{O} W-\mathrm{H} 2 W \cdots \mathrm{C} 11^{\text {iii }}$ & $0.89(4)$ & $2.37(4)$ & $3.183(3)$ & $151(7)$ \\
$\mathrm{O}^{2} W-\mathrm{H} 1 W 2 \cdots \mathrm{O} 1 W$ & $0.91(7)$ & $1.96(7)$ & $2.857(5)$ & $169(6)$ \\
$\mathrm{O}^{2} W-\mathrm{H} 2 W 2 \cdots \mathrm{Cl} 1^{\text {iv }}$ & $0.89(5)$ & $2.40(5)$ & $3.215(4)$ & $153(5)$ \\
\hline
\end{tabular}

Symmetry codes: (i) $-x+\frac{1}{2}, y+\frac{1}{2},-z+\frac{1}{2} ; \quad$ (ii) $\quad-x+1,-y+1,-z+1$; (iii) $-x+\frac{1}{2}, y-\frac{1}{2},-z+\frac{3}{2}$; (iv) $x, y-1, z$.

(Tashkhodzhaev et al., 1995; Turgunov et al., 1998, 2003). The above mentioned $\mathrm{N}-\mathrm{H} \cdots \mathrm{N}$ hydrogen bonds link the molecules into a three-dimensional network. The crystal structure of III is stabilized by $\pi-\pi$ interactions [centroid-centroid distances in the range 3.4113 (16)-3.9080 (18) А] .

\section{Database survey}

A search of the Cambridge Structural Database (CSD, version 5.41, including the update of January 2020; Groom et al., 2016) confirmed that three related compounds had been structurally characterized in which the benzene ring of the quinazolin4(3H)-ones contains a nitro group [refcodes GAPPUK (Yu et al., 2012), GISXOW (Yong et al., 2008) and RUGKEK (Wu et al., 2009)].

The crystal structures of quinazolin-4(3H)-one and its first metal coordination compound have also been reported [BIHJIO (Liao et al., 2018) and NALFEN (Turgunov \& Englert, 2010)].

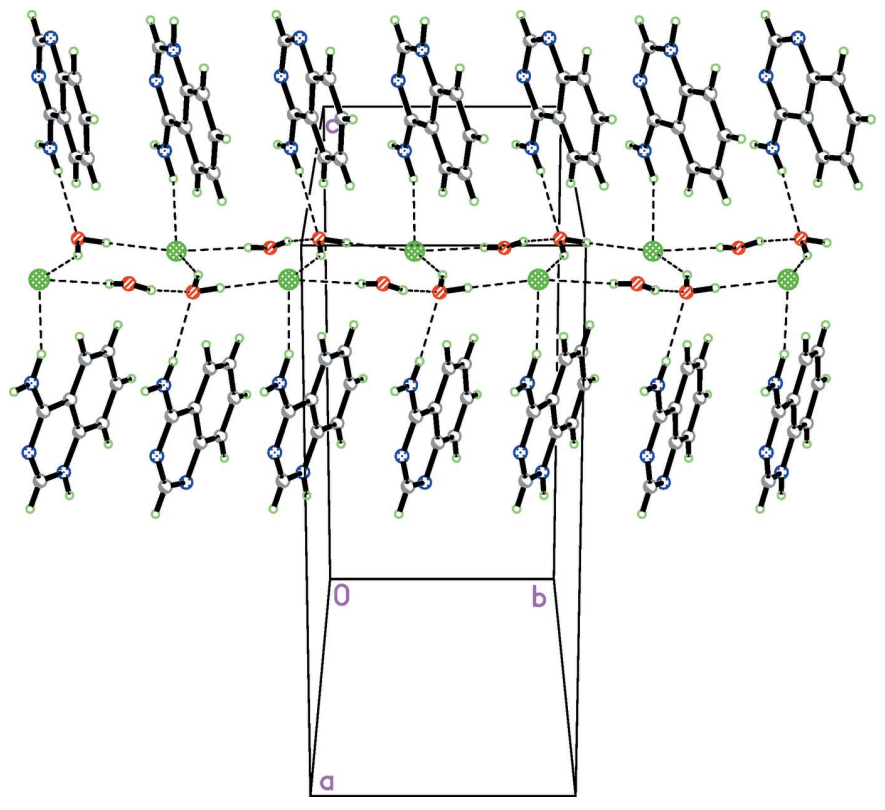

Figure 6

Part of the crystal structure of III showing the hydrogen-bonding scheme. Colour code: $\mathrm{C}$ grey, $\mathrm{H}$ light green, $\mathrm{Cl}$ bright green, $\mathrm{N}$ blue, $\mathrm{O}$ red.

\section{Synthesis and crystallization}

Compound I: In a three-necked flask equipped with a mechanical stirrer and reflux condenser, quinazolin-4(3H)one $(22.5 \mathrm{~g}, 0.15 \mathrm{~mol})$ was dissolved in $78 \mathrm{ml}$ of concentrated sulfuric acid at $303 \mathrm{~K}$ for $1 \mathrm{~h}$. Then a nitrating mixture $(21 \mathrm{ml}$ of nitric acid and $18 \mathrm{ml}$ of concentrated sulfuric acid) was added to the flask under vigorous stirring of the mixture. The reaction mixture was stirred for another hour, maintaining a temperature not higher than $303 \mathrm{~K}$, and then for another hour at room temperature. At room temperature, $45 \mathrm{ml}$ of nitric acid were added dropwise to the reaction mixture over a period of $1 \mathrm{~h}$. The reaction mixture was left at room temperature for $10 \mathrm{~h}$. The contents of the flask were poured into a dish containing ice, the resulting precipitate was filtered off, washed with water and dried and recrystallized from ethanol to obtain $25.7 \mathrm{~g}$ of pure compound $\mathbf{I}$ as single crystals in $87.4 \%$ yield, m.p. 560-562 K.

Compound II: In a three-necked flask equipped with a mechanical stirrer and reflux condenser, $12.6 \mathrm{~g}(56 \mathrm{mmol})$ of tin (II) chloride dihydrate $\left(\mathrm{SnCl}_{2} \cdot 2 \mathrm{H}_{2} \mathrm{O}\right)$ were cooled in an ice bath and $16.98 \mathrm{ml}$ of concentrated $(36 \%) \mathrm{HCl}$ were added, then $3 \mathrm{~g}$ ( $16 \mathrm{mmol})$ of quinazolin-4-one as a suspension in $20 \mathrm{ml}$ of ethanol and $7 \mathrm{ml}$ of $\mathrm{HCl}(36 \%)$ were added portionwise with stirring of the mixture. The reaction was carried out for $10-15$ minutes at $\sim 273 \mathrm{~K}, 30 \mathrm{~min}$ at room temperature and $2 \mathrm{~h}$ in a water bath $(\sim 363 \mathrm{~K})$. The reaction mixture was left overnight at room temperature, diluted with water, and brought to a strongly alkaline medium $(\mathrm{pH}=10$ 11) with $10 \%$ of sodium hydroxide, in which the expected 6 amino-3N-quinazoline-4-one was dissolved, so that the chloride was brought to a neutral medium in the presence of acid, and precipitated when converted to an alkaline medium with ammonia. The precipitate was filtered, washed with water until it reached a neutral medium, and dried at room temperature. The precipitate was recrystallized from ethanol and $6.67 \mathrm{~g}$ of pure compound II were obtained representing an $88.1 \%$ yield, m.p. 589-591 K.

Compound III: Crystals of compound III were obtained as a minor additional product in the reaction of 4-chloroquinazoline with ammonia.

\section{Refinement}

Crystal data, data collection and structure refinement details are summarized in Table 7. C-bound $\mathrm{H}$ atoms were placed in calculated positions and refined to ride on their parent atoms: $\mathrm{C}-\mathrm{H}=0.93 \AA$ with $U_{\text {iso }}(\mathrm{H})=1.2 U_{\text {eq }}(\mathrm{C})$. Hydrogen atoms of the water molecules and those bonded to nitrogen atoms were located in electron density difference maps and were freely refined.

\section{Acknowledgements}

X-ray diffraction studies were performed at the Centre of Collective Usage of Equipment of the Institute of Bioorganic Chemistry of the Uzbekistan Academy of Sciences. Professor Bakhtiyar Ibragimov is acknowledged for support with the diffraction measurements. 
Table 7

Experimental details.

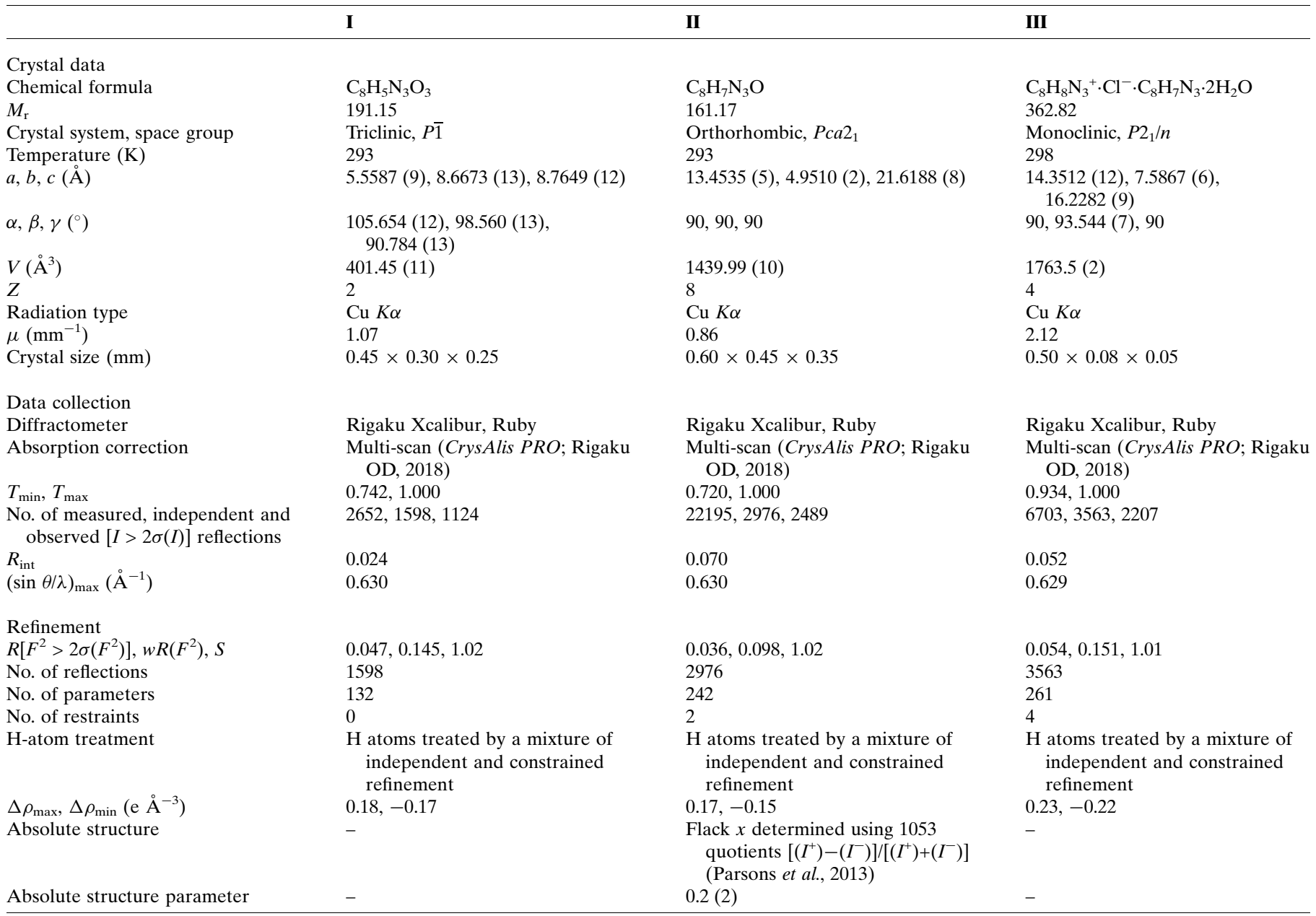

Computer programs: CrysAlis PRO (Rigaku OD, 2018), SHELXT2018/2 (Sheldrick, 2015a), SHELXL2014/7 (Sheldrick, 2015b), XP in SHELXTL (Sheldrick, 2008) and publCIF (Westrip, 2010).

\section{References}

Alagarsamy, V., Solomon, V. R. \& Dhanabal, K. (2007). Bioorg. Med. Chem. 15, 235-241.

Antipenko, L., Karpenko, A., Kovalenko, S., Katsev, A., Komarovska-Porokhnyavets, E., Novikov, V. \& Chekotilo, A. (2009). Chem. Pharm. Bull. 57, 580-585.

Azab, A., Nassar, A. \& Azab, A. N. (2016). Molecules, 21, 1321-1340. Chandregowda, V., Kush, A. K. \& Chandrasekara Reddy, G. (2009). Eur. J. Med. Chem. 44, 3046-3055.

De Clercq, E. \& Field, H. J. (2006). Br. J. Pharmacol. 147, 1-11.

Ding, Q., Chichak, K. \& Lown, J. W. (1999). Curr. Med. Chem. 6, 1-27.

Groom, C. R., Bruno, I. J., Lightfoot, M. P. \& Ward, S. C. (2016). Acta Cryst. B72, 171-179.

Hess, H. J., Cronin, T. H. \& Scriabine, A. (1968). J. Med. Chem. 11, 130-136.

Ishikawa, H., Colby, D. A., Seto, S., Va, P., Tam, A., Kakei, H., Rayl, T. J., Hwang, I. \& Boger, D. L. (2009). J. Am. Chem. Soc. 131, 49044916.

Liao, B.-L., Pan, Y.-J., Zhang, W. \& Pan, L.-W. (2018). Chem. Biodivers. 15, e1800152.

Nandy, P., Vishalakshi, M. T. \& Bhat, A. R. (2006). Indian J. Heterocycl. Chem. 15, 293-294.

Paneersalvam, P., Raj, T., Ishar, M. P. S., Singh, B., Sharma, V. \& Rather, B. A. (2010). Indian J. Pharm. Sci. 72, 375-378.
Parsons, S., Flack, H. D. \& Wagner, T. (2013). Acta Cryst. B69, 249259.

Perumalla, S. R., Pedireddi, V. R. \& Sun, C. C. (2013). Cryst. Growth Des. 13, 429-432.

Rigaku OD (2018). CrysAlis PRO. Rigaku Oxford Diffraction, Yarnton, England.

Sheldrick, G. M. (2008). Acta Cryst. A64, 112-122.

Sheldrick, G. M. (2015a). Acta Cryst. A71, 3-8.

Sheldrick, G. M. (2015b). Acta Cryst. C71, 3-8.

Tashkhodzhaev, B., Molchanov, L. V., Turgunov, K. K., Makhmudov, M. K. \& Aripov, Kh. N. (1995). Chem. Nat. Compd. 31, 349-352.

Turgunov, K. \& Englert, U. (2010). Acta Cryst. E66, m1457.

Turgunov, K. K., Tashkhodzhaev, B., Molchanov, L. V., Musaeva, G. V. \& Aripov, Kh. N. (1998). Chem. Nat. Compd. 34, 300-303.

Turgunov, K. K., Tashkhodzhaev, B., Molchanov, L. V. \& Shakhidoyatov, Kh. M. (2003). Chem. Nat. Compd. 39, 379-382.

Westrip, S. P. (2010). J. Appl. Cryst. 43, 920-925.

Wu, Y., Ji, A., Zhang, A. \& Shen, Y. (2009). Acta Cryst. E65, 03075.

Yong, J.-P., Yu, G.-P., Li, J.-M., Hou, X.-L. \& Aisa, H. A. (2008). Acta Cryst. E64, o427.

Yu, Y. (2012). Acta Cryst. E68, o304. 


\section{supporting information}

Acta Cryst. (2021). E77, 989-993 [https://doi.org/10.1107/S2056989021008823]

Crystal structures of 6-nitroquinazolin-4(3H)-one, 6-aminoquinazolin-4(3H)one and 4-aminoquinazoline hemihydrochloride dihydrate

\section{Kambarali Turgunov, Mirjalol Ziyadullaev, Farkhod Khoshimov, Rikhsiboy Karimov and Burkhon Elmuradov}

\section{Computing details}

For all structures, data collection: CrysAlis PRO (Rigaku OD, 2018); cell refinement: CrysAlis PRO (Rigaku OD, 2018); data reduction: CrysAlis PRO (Rigaku OD, 2018); program(s) used to solve structure: SHELXT2018/2 (Sheldrick, 2015a); program(s) used to refine structure: SHELXL2014/7 (Sheldrick, 2015b); molecular graphics: XP in SHELXTL (Sheldrick, 2008); software used to prepare material for publication: publCIF (Westrip, 2010).

6-Nitroquinazolin-4(3H)-one (I)

Crystal data

$\mathrm{C}_{8} \mathrm{H}_{5} \mathrm{~N}_{3} \mathrm{O}_{3}$

$M_{r}=191.15$

Triclinic, $P \overline{1}$

$a=5.5587$ (9) $\AA$

$b=8.6673(13) \AA$

$c=8.7649(12) \AA$

$\alpha=105.654(12)^{\circ}$

$\beta=98.560(13)^{\circ}$

$\gamma=90.784(13)^{\circ}$

$V=401.45(11) \AA^{3}$

$Z=2$

\section{Data collection}

Rigaku Xcalibur, Ruby diffractometer

Radiation source: Enhance $(\mathrm{Cu}) \mathrm{X}$-ray Source Graphite monochromator

Detector resolution: 10.2576 pixels $\mathrm{mm}^{-1}$ $\omega$ scans

Absorption correction: multi-scan

(CrysAlisPro; Rigaku OD, 2018)

$T_{\min }=0.742, T_{\max }=1.000$

\section{Refinement}

Refinement on $F^{2}$

Least-squares matrix: full

$R\left[F^{2}>2 \sigma\left(F^{2}\right)\right]=0.047$

$w R\left(F^{2}\right)=0.145$

$S=1.02$

1598 reflections
$F(000)=196$

$D_{\mathrm{x}}=1.581 \mathrm{Mg} \mathrm{m}^{-3}$

Melting point: $560(2) \mathrm{K}$

$\mathrm{Cu} K \alpha$ radiation, $\lambda=1.54184 \AA$

Cell parameters from 950 reflections

$\theta=5.3-74.5^{\circ}$

$\mu=1.07 \mathrm{~mm}^{-1}$

$T=293 \mathrm{~K}$

Prism, colourless

$0.45 \times 0.30 \times 0.25 \mathrm{~mm}$

2652 measured reflections

1598 independent reflections

1124 reflections with $I>2 \sigma(I)$

$R_{\text {int }}=0.024$

$\theta_{\text {max }}=76.3^{\circ}, \theta_{\min }=5.3^{\circ}$

$h=-6 \rightarrow 7$

$k=-7 \rightarrow 10$

$l=-10 \rightarrow 9$

132 parameters

0 restraints

Primary atom site location: structure-invariant direct methods

Hydrogen site location: mixed 
$\mathrm{H}$ atoms treated by a mixture of independent and constrained refinement

$w=1 /\left[\sigma^{2}\left(F_{\mathrm{o}}^{2}\right)+(0.0615 P)^{2}\right]$

where $P=\left(F_{\mathrm{o}}^{2}+2 F_{\mathrm{c}}^{2}\right) / 3$

$(\Delta / \sigma)_{\max }<0.001$

$\Delta \rho_{\max }=0.18$ e $\AA^{-3}$

Special details

Geometry. All esds (except the esd in the dihedral angle between two 1.s. planes) are estimated using the full covariance matrix. The cell esds are taken into account individually in the estimation of esds in distances, angles and torsion angles; correlations between esds in cell parameters are only used when they are defined by crystal symmetry. An approximate (isotropic) treatment of cell esds is used for estimating esds involving l.s. planes.

Fractional atomic coordinates and isotropic or equivalent isotropic displacement parameters $\left(\AA^{2}\right)$

\begin{tabular}{lllll}
\hline & $x$ & $y$ & $z$ & $U_{\text {iso }} / U_{\text {eq }}$ \\
\hline O1 & $0.6353(3)$ & $0.19086(17)$ & $0.52777(19)$ & $0.0644(5)$ \\
O2 & $0.7328(5)$ & $0.9420(2)$ & $0.8525(3)$ & $0.0988(8)$ \\
O3 & $0.8935(3)$ & $0.7763(2)$ & $0.6717(2)$ & $0.0739(5)$ \\
N1 & $0.1305(3)$ & $0.3183(2)$ & $0.8279(2)$ & $0.0587(5)$ \\
C2 & $0.1601(4)$ & $0.1736(3)$ & $0.7482(3)$ & $0.0585(5)$ \\
H2 & 0.0576 & 0.0929 & 0.7592 & $0.070^{*}$ \\
N3 & $0.3277(4)$ & $0.1287(2)$ & $0.6497(2)$ & $0.0566(5)$ \\
C4 & $0.4881(4)$ & $0.2343(2)$ & $0.6209(2)$ & $0.0517(5)$ \\
C4A & $0.4645(4)$ & $0.4012(2)$ & $0.7094(2)$ & $0.0476(5)$ \\
C5 & $0.6197(4)$ & $0.5228(2)$ & $0.6955(3)$ & $0.0519(5)$ \\
H5 & 0.7399 & 0.4998 & 0.6306 & $0.062^{*}$ \\
C6 & $0.5897(4)$ & $0.6775(2)$ & $0.7807(3)$ & $0.0529(5)$ \\
C7 & $0.4120(4)$ & $0.7162(2)$ & $0.8796(3)$ & $0.0569(5)$ \\
H7 & 0.3956 & 0.8224 & 0.9349 & $0.068^{*}$ \\
C8 & $0.2626(4)$ & $0.5968(2)$ & $0.8944(3)$ & $0.0567(5)$ \\
H8 & 0.1446 & 0.6214 & 0.9607 & $0.068^{*}$ \\
C8A & $0.2866(4)$ & $0.4359(2)$ & $0.8092(2)$ & $0.0507(5)$ \\
N9 & $0.7504(4)$ & $0.8071(2)$ & $0.7678(3)$ & $0.0635(5)$ \\
H3 & $0.334(5)$ & $0.038(3)$ & $0.598(3)$ & $0.057(6)^{*}$ \\
\end{tabular}

Atomic displacement parameters $\left(\AA^{2}\right)$

\begin{tabular}{lllllll}
\hline & $U^{11}$ & $U^{22}$ & $U^{33}$ & $U^{12}$ & $U^{13}$ & $U^{23}$ \\
\hline O1 & $0.0731(10)$ & $0.0452(8)$ & $0.0736(11)$ & $0.0019(7)$ & $0.0352(9)$ & $0.0014(7)$ \\
O2 & $0.1272(18)$ & $0.0417(9)$ & $0.1230(17)$ & $-0.0165(10)$ & $0.0525(15)$ & $-0.0014(9)$ \\
O3 & $0.0730(11)$ & $0.0618(10)$ & $0.0918(13)$ & $-0.0050(8)$ & $0.0287(10)$ & $0.0215(9)$ \\
N1 & $0.0605(10)$ & $0.0490(9)$ & $0.0681(11)$ & $-0.0004(7)$ & $0.0260(9)$ & $0.0102(8)$ \\
C2 & $0.0621(12)$ & $0.0457(11)$ & $0.0690(14)$ & $-0.0031(9)$ & $0.0213(11)$ & $0.0126(9)$ \\
N3 & $0.0674(11)$ & $0.0368(8)$ & $0.0646(11)$ & $0.0004(7)$ & $0.0226(9)$ & $0.0058(8)$ \\
C4 & $0.0553(11)$ & $0.0431(10)$ & $0.0557(11)$ & $0.0035(8)$ & $0.0169(9)$ & $0.0073(8)$ \\
C4A & $0.0534(10)$ & $0.0398(9)$ & $0.0486(10)$ & $0.0030(8)$ & $0.0129(8)$ & $0.0082(8)$ \\
C5 & $0.0549(11)$ & $0.0478(11)$ & $0.0542(11)$ & $0.0042(8)$ & $0.0154(9)$ & $0.0122(8)$ \\
C6 & $0.0571(11)$ & $0.0430(10)$ & $0.0579(11)$ & $-0.0013(8)$ & $0.0111(9)$ & $0.0118(8)$ \\
C7 & $0.0674(13)$ & $0.0396(10)$ & $0.0600(12)$ & $0.0067(9)$ & $0.0143(10)$ & $0.0052(8)$
\end{tabular}


supporting information

\begin{tabular}{lllllll} 
C8 & $0.0617(12)$ & $0.0464(11)$ & $0.0606(12)$ & $0.0070(9)$ & $0.0211(10)$ & $0.0061(9)$ \\
C8A & $0.0535(11)$ & $0.0436(10)$ & $0.0542(11)$ & $0.0027(8)$ & $0.0135(9)$ & $0.0092(8)$ \\
N9 & $0.0694(12)$ & $0.0453(10)$ & $0.0744(12)$ & $-0.0043(8)$ & $0.0133(10)$ & $0.0138(8)$ \\
\hline
\end{tabular}

Geometric parameters $\left(\AA,{ }^{o}\right)$

\begin{tabular}{|c|c|c|c|}
\hline $\mathrm{O} 1-\mathrm{C} 4$ & $1.233(2)$ & $\mathrm{C} 4 \mathrm{~A}-\mathrm{C} 5$ & $1.395(3)$ \\
\hline $\mathrm{O} 2-\mathrm{N} 9$ & $1.218(2)$ & $\mathrm{C} 4 \mathrm{~A}-\mathrm{C} 8 \mathrm{~A}$ & $1.399(3)$ \\
\hline $\mathrm{O} 3-\mathrm{N} 9$ & $1.223(2)$ & $\mathrm{C} 5-\mathrm{C} 6$ & $1.374(3)$ \\
\hline $\mathrm{N} 1-\mathrm{C} 2$ & $1.287(3)$ & $\mathrm{C} 5-\mathrm{H} 5$ & 0.9300 \\
\hline $\mathrm{N} 1-\mathrm{C} 8 \mathrm{~A}$ & $1.388(3)$ & $\mathrm{C} 6-\mathrm{C} 7$ & $1.395(3)$ \\
\hline $\mathrm{C} 2-\mathrm{N} 3$ & $1.354(3)$ & $\mathrm{C} 6-\mathrm{N} 9$ & $1.464(3)$ \\
\hline $\mathrm{C} 2-\mathrm{H} 2$ & 0.9300 & $\mathrm{C} 7-\mathrm{C} 8$ & $1.363(3)$ \\
\hline $\mathrm{N} 3-\mathrm{C} 4$ & $1.366(3)$ & $\mathrm{C} 7-\mathrm{H} 7$ & 0.9300 \\
\hline N3-H3 & $0.80(3)$ & $\mathrm{C} 8-\mathrm{C} 8 \mathrm{~A}$ & $1.412(3)$ \\
\hline $\mathrm{C} 4-\mathrm{C} 4 \mathrm{~A}$ & $1.463(3)$ & $\mathrm{C} 8-\mathrm{H} 8$ & 0.9300 \\
\hline $\mathrm{C} 2-\mathrm{N} 1-\mathrm{C} 8 \mathrm{~A}$ & $115.80(17)$ & $\mathrm{C} 5-\mathrm{C} 6-\mathrm{C} 7$ & $122.54(19)$ \\
\hline $\mathrm{N} 1-\mathrm{C} 2-\mathrm{N} 3$ & $125.57(19)$ & $\mathrm{C} 5-\mathrm{C} 6-\mathrm{N} 9$ & $118.89(18)$ \\
\hline $\mathrm{N} 1-\mathrm{C} 2-\mathrm{H} 2$ & 117.2 & $\mathrm{C} 7-\mathrm{C} 6-\mathrm{N} 9$ & $118.57(18)$ \\
\hline $\mathrm{N} 3-\mathrm{C} 2-\mathrm{H} 2$ & 117.2 & $\mathrm{C} 8-\mathrm{C} 7-\mathrm{C} 6$ & $119.31(18)$ \\
\hline $\mathrm{C} 2-\mathrm{N} 3-\mathrm{C} 4$ & $123.57(17)$ & $\mathrm{C} 8-\mathrm{C} 7-\mathrm{H} 7$ & 120.3 \\
\hline $\mathrm{C} 2-\mathrm{N} 3-\mathrm{H} 3$ & $122.0(18)$ & $\mathrm{C} 6-\mathrm{C} 7-\mathrm{H} 7$ & 120.3 \\
\hline $\mathrm{C} 4-\mathrm{N} 3-\mathrm{H} 3$ & $114.3(18)$ & $\mathrm{C} 7-\mathrm{C} 8-\mathrm{C} 8 \mathrm{~A}$ & $120.21(19)$ \\
\hline $\mathrm{O} 1-\mathrm{C} 4-\mathrm{N} 3$ & $122.34(17)$ & $\mathrm{C} 7-\mathrm{C} 8-\mathrm{H} 8$ & 119.9 \\
\hline $\mathrm{O} 1-\mathrm{C} 4-\mathrm{C} 4 \mathrm{~A}$ & $124.23(18)$ & $\mathrm{C} 8 \mathrm{~A}-\mathrm{C} 8-\mathrm{H} 8$ & 119.9 \\
\hline $\mathrm{N} 3-\mathrm{C} 4-\mathrm{C} 4 \mathrm{~A}$ & $113.43(16)$ & $\mathrm{N} 1-\mathrm{C} 8 \mathrm{~A}-\mathrm{C} 4 \mathrm{~A}$ & $122.73(18)$ \\
\hline $\mathrm{C} 5-\mathrm{C} 4 \mathrm{~A}-\mathrm{C} 8 \mathrm{~A}$ & $120.89(18)$ & $\mathrm{N} 1-\mathrm{C} 8 \mathrm{~A}-\mathrm{C} 8$ & $118.18(18)$ \\
\hline $\mathrm{C} 5-\mathrm{C} 4 \mathrm{~A}-\mathrm{C} 4$ & $120.21(17)$ & $\mathrm{C} 4 \mathrm{~A}-\mathrm{C} 8 \mathrm{~A}-\mathrm{C} 8$ & $119.09(19)$ \\
\hline $\mathrm{C} 8 \mathrm{~A}-\mathrm{C} 4 \mathrm{~A}-\mathrm{C} 4$ & $118.90(18)$ & $\mathrm{O} 2-\mathrm{N} 9-\mathrm{O} 3$ & $122.9(2)$ \\
\hline $\mathrm{C} 6-\mathrm{C} 5-\mathrm{C} 4 \mathrm{~A}$ & $117.95(18)$ & $\mathrm{O} 2-\mathrm{N} 9-\mathrm{C} 6$ & $118.0(2)$ \\
\hline $\mathrm{C} 6-\mathrm{C} 5-\mathrm{H} 5$ & 121.0 & $\mathrm{O} 3-\mathrm{N} 9-\mathrm{C} 6$ & $119.03(18)$ \\
\hline $\mathrm{C} 4 \mathrm{~A}-\mathrm{C} 5-\mathrm{H} 5$ & 121.0 & & \\
\hline $\mathrm{C} 8 \mathrm{~A}-\mathrm{N} 1-\mathrm{C} 2-\mathrm{N} 3$ & $-0.1(4)$ & $\mathrm{C} 6-\mathrm{C} 7-\mathrm{C} 8-\mathrm{C} 8 \mathrm{~A}$ & $-0.5(4)$ \\
\hline $\mathrm{N} 1-\mathrm{C} 2-\mathrm{N} 3-\mathrm{C} 4$ & $-0.7(4)$ & $\mathrm{C} 2-\mathrm{N} 1-\mathrm{C} 8 \mathrm{~A}-\mathrm{C} 4 \mathrm{~A}$ & $0.5(3)$ \\
\hline $\mathrm{C} 2-\mathrm{N} 3-\mathrm{C} 4-\mathrm{O} 1$ & $-178.3(2)$ & $\mathrm{C} 2-\mathrm{N} 1-\mathrm{C} 8 \mathrm{~A}-\mathrm{C} 8$ & $-179.8(2)$ \\
\hline $\mathrm{C} 2-\mathrm{N} 3-\mathrm{C} 4-\mathrm{C} 4 \mathrm{~A}$ & $1.0(3)$ & $\mathrm{C} 5-\mathrm{C} 4 \mathrm{~A}-\mathrm{C} 8 \mathrm{~A}-\mathrm{N} 1$ & $-179.2(2)$ \\
\hline $\mathrm{O} 1-\mathrm{C} 4-\mathrm{C} 4 \mathrm{~A}-\mathrm{C} 5$ & $-2.2(3)$ & $\mathrm{C} 4-\mathrm{C} 4 \mathrm{~A}-\mathrm{C} 8 \mathrm{~A}-\mathrm{N} 1$ & $0.0(3)$ \\
\hline $\mathrm{N} 3-\mathrm{C} 4-\mathrm{C} 4 \mathrm{~A}-\mathrm{C} 5$ & $178.5(2)$ & $\mathrm{C} 5-\mathrm{C} 4 \mathrm{~A}-\mathrm{C} 8 \mathrm{~A}-\mathrm{C} 8$ & $1.1(3)$ \\
\hline $\mathrm{O} 1-\mathrm{C} 4-\mathrm{C} 4 \mathrm{~A}-\mathrm{C} 8 \mathrm{~A}$ & $178.6(2)$ & $\mathrm{C} 4-\mathrm{C} 4 \mathrm{~A}-\mathrm{C} 8 \mathrm{~A}-\mathrm{C} 8$ & $-179.8(2)$ \\
\hline $\mathrm{N} 3-\mathrm{C} 4-\mathrm{C} 4 \mathrm{~A}-\mathrm{C} 8 \mathrm{~A}$ & $-0.7(3)$ & $\mathrm{C} 7-\mathrm{C} 8-\mathrm{C} 8 \mathrm{~A}-\mathrm{N} 1$ & $180.0(2)$ \\
\hline $\mathrm{C} 8 \mathrm{~A}-\mathrm{C} 4 \mathrm{~A}-\mathrm{C} 5-\mathrm{C} 6$ & $-1.0(3)$ & $\mathrm{C} 7-\mathrm{C} 8-\mathrm{C} 8 \mathrm{~A}-\mathrm{C} 4 \mathrm{~A}$ & $-0.2(3)$ \\
\hline $\mathrm{C} 4-\mathrm{C} 4 \mathrm{~A}-\mathrm{C} 5-\mathrm{C} 6$ & $179.8(2)$ & $\mathrm{C} 5-\mathrm{C} 6-\mathrm{N} 9-\mathrm{O} 2$ & $-175.0(2)$ \\
\hline $\mathrm{C} 4 \mathrm{~A}-\mathrm{C} 5-\mathrm{C} 6-\mathrm{C} 7$ & $0.3(3)$ & $\mathrm{C} 7-\mathrm{C} 6-\mathrm{N} 9-\mathrm{O} 2$ & $4.9(4)$ \\
\hline $\mathrm{C} 4 \mathrm{~A}-\mathrm{C} 5-\mathrm{C} 6-\mathrm{N} 9$ & $-179.9(2)$ & $\mathrm{C} 5-\mathrm{C} 6-\mathrm{N} 9-\mathrm{O} 3$ & $6.0(3)$ \\
\hline $\mathrm{C} 5-\mathrm{C} 6-\mathrm{C} 7-\mathrm{C} 8$ & $0.5(4)$ & $\mathrm{C} 7-\mathrm{C} 6-\mathrm{N} 9-\mathrm{O} 3$ & $-174.2(2)$ \\
\hline $\mathrm{N} 9-\mathrm{C} 6-\mathrm{C} 7-\mathrm{C} 8$ & $-179.3(2)$ & & \\
\hline
\end{tabular}


Hydrogen-bond geometry $\left(\AA,{ }^{\circ}\right)$

\begin{tabular}{lllll}
\hline$D-\mathrm{H} \cdots A$ & $D-\mathrm{H}$ & $\mathrm{H} \cdots A$ & $D \cdots A$ & $D-\mathrm{H} \cdots A$ \\
\hline $\mathrm{N} 3-\mathrm{H} 3 \cdots \mathrm{O} 1^{\mathrm{i}}$ & $0.80(3)$ & $2.02(3)$ & $2.814(2)$ & $178(4)$ \\
$\mathrm{C} 8-\mathrm{H} 8 \cdots \mathrm{N} 1^{\mathrm{ii}}$ & 0.93 & 2.53 & $3.450(3)$ & 172 \\
$\mathrm{C} 2-\mathrm{H} 2 \cdots \mathrm{O} 2^{\mathrm{iii}}$ & 0.93 & 2.57 & $3.466(4)$ & 163 \\
$\mathrm{C} 7-\mathrm{H} 7 \cdots \mathrm{O} 2^{\mathrm{iv}}$ & 0.93 & 2.56 & $3.437(3)$ & 158
\end{tabular}

Symmetry codes: (i) $-x+1,-y,-z+1$; (ii) $-x,-y+1,-z+2$; (iii) $x-1, y-1, z$; (iv) $-x+1,-y+2,-z+2$.

6-Aminoquinazolin-4(3H)-one (II)

Crystal data

\section{$\mathrm{C}_{8} \mathrm{H}_{7} \mathrm{~N}_{3} \mathrm{O}$}

$M_{r}=161.17$

Orthorhombic, $P_{c a 2_{1}}$

$a=13.4535$ (5) $\AA$

$b=4.9510(2) \AA$

$c=21.6188(8) \AA$

$V=1439.99(10) \AA^{3}$

$Z=8$

$F(000)=672$

\section{Data collection}

Rigaku Xcalibur, Ruby diffractometer

Radiation source: Enhance $(\mathrm{Cu}) \mathrm{X}$-ray Source

Graphite monochromator

Detector resolution: 10.2576 pixels $\mathrm{mm}^{-1}$

$\omega$ scans

Absorption correction: multi-scan

(CrysAlisPro; Rigaku OD, 2018)

$T_{\min }=0.720, T_{\max }=1.000$

\section{Refinement}

Refinement on $F^{2}$

Least-squares matrix: full

$R\left[F^{2}>2 \sigma\left(F^{2}\right)\right]=0.036$

$w R\left(F^{2}\right)=0.098$

$S=1.02$

2976 reflections

242 parameters

2 restraints

Primary atom site location: structure-invariant direct methods

Hydrogen site location: mixed

$\mathrm{H}$ atoms treated by a mixture of independent and constrained refinement
$D_{\mathrm{x}}=1.487 \mathrm{Mg} \mathrm{m}^{-3}$

Melting point: $589(2) \mathrm{K}$

$\mathrm{Cu} K \alpha$ radiation, $\lambda=1.54184 \AA$

Cell parameters from 5076 reflections

$\theta=4.1-75.8^{\circ}$

$\mu=0.86 \mathrm{~mm}^{-1}$

$T=293 \mathrm{~K}$

Prism, colourless

$0.60 \times 0.45 \times 0.35 \mathrm{~mm}$

22195 measured reflections

2976 independent reflections

2489 reflections with $I>2 \sigma(I)$

$R_{\text {int }}=0.070$

$\theta_{\max }=76.1^{\circ}, \theta_{\min }=4.1^{\circ}$

$h=-16 \rightarrow 16$

$k=-6 \rightarrow 6$

$l=-26 \rightarrow 27$ $w=1 /\left[\sigma^{2}\left(F_{\mathrm{o}}^{2}\right)+(0.0516 P)^{2}+0.144 P\right]$ where $P=\left(F_{\mathrm{o}}{ }^{2}+2 F_{\mathrm{c}}{ }^{2}\right) / 3$

$(\Delta / \sigma)_{\max }=0.001$

$\Delta \rho_{\max }=0.17$ e $\AA^{-3}$

$\Delta \rho_{\min }=-0.14$ e $\AA^{-3}$

Extinction correction: SHELXL-2014/7

(Sheldrick, 2015), $\mathrm{Fc}^{*}=\mathrm{kFc}\left[1+0.001 \times \mathrm{Fc}^{2} \lambda^{3} / \sin (2 \theta)\right]^{-1 / 4}$

Extinction coefficient: 0.0034 (4)

Absolute structure: Flack $x$ determined using 1053 quotients $\left[\left(I^{+}\right)-\left(I^{-}\right)\right] /\left[\left(I^{+}\right)+\left(I^{-}\right)\right]$(Parsons et al., 2013)

Absolute structure parameter: 0.2 (2)

\section{Special details}

Geometry. All esds (except the esd in the dihedral angle between two 1.s. planes) are estimated using the full covariance matrix. The cell esds are taken into account individually in the estimation of esds in distances, angles and torsion angles; correlations between esds in cell parameters are only used when they are defined by crystal symmetry. An approximate (isotropic) treatment of cell esds is used for estimating esds involving l.s. planes. 
Fractional atomic coordinates and isotropic or equivalent isotropic displacement parameters $\left(\AA^{2}\right)$

\begin{tabular}{|c|c|c|c|c|}
\hline & $x$ & $y$ & $z$ & $U_{\text {iso }} * / U_{\text {eq }}$ \\
\hline $\mathrm{O} 1 \mathrm{~A}$ & $0.40087(16)$ & $0.0190(4)$ & $0.46170(11)$ & $0.0394(5)$ \\
\hline N1A & $0.6791(2)$ & $0.2101(6)$ & $0.39888(13)$ & $0.0427(7)$ \\
\hline $\mathrm{C} 2 \mathrm{~A}$ & $0.6607(2)$ & $0.0205(7)$ & $0.43824(16)$ & $0.0408(8)$ \\
\hline $\mathrm{H} 2 \mathrm{~A}$ & 0.7142 & -0.0786 & 0.4532 & $0.049 *$ \\
\hline N3A & $0.5680(2)$ & $-0.0455(6)$ & $0.45958(13)$ & $0.0373(6)$ \\
\hline $\mathrm{C} 4 \mathrm{~A}$ & $0.4828(2)$ & $0.0850(6)$ & $0.44094(14)$ & $0.0337(7)$ \\
\hline C4AA & $0.4994(2)$ & $0.3010(6)$ & $0.39636(14)$ & $0.0338(7)$ \\
\hline C5A & $0.4197(2)$ & $0.4509(6)$ & $0.37325(14)$ & $0.0361(7)$ \\
\hline $\mathrm{H} 5 \mathrm{~A}$ & 0.3555 & 0.4132 & 0.3867 & $0.043^{*}$ \\
\hline C6A & $0.4353(2)$ & $0.6566(7)$ & $0.33025(15)$ & $0.0368(7)$ \\
\hline C7A & $0.5343(3)$ & $0.7079(7)$ & $0.31113(15)$ & $0.0394(8)$ \\
\hline H7A & 0.5464 & 0.8449 & 0.2827 & $0.047 *$ \\
\hline C8A & $0.6122(3)$ & $0.5608(7)$ & $0.33352(16)$ & $0.0412(8)$ \\
\hline H8A & 0.6763 & 0.5982 & 0.3198 & $0.049 *$ \\
\hline C8AA & 0.5971 & $0.3541(6)$ & $0.37691(15)$ & $0.0364(7)$ \\
\hline N9A & 0.3578 & $0.8001(6)$ & $0.30523(15)$ & $0.0469(8)$ \\
\hline O1B & $0.53939(17)$ & $0.5050(4)$ & $0.53859(12)$ & $0.0403(6)$ \\
\hline N1B & $0.2624(2)$ & $0.3084(6)$ & $0.60178(14)$ & $0.0451(7)$ \\
\hline $\mathrm{C} 2 \mathrm{~B}$ & $0.2799(2)$ & $0.4952(7)$ & $0.56163(16)$ & $0.0430(8)$ \\
\hline $\mathrm{H} 2 \mathrm{~B}$ & 0.2257 & 0.5891 & 0.5458 & $0.052 *$ \\
\hline N3B & $0.3720(2)$ & $0.5655(5)$ & $0.54065(13)$ & $0.0385(6)$ \\
\hline C4B & $0.4570(3)$ & $0.4384(6)$ & $0.55929(14)$ & $0.0332(7)$ \\
\hline $\mathrm{C} 4 \mathrm{AB}$ & $0.4421(2)$ & $0.2222(6)$ & $0.60434(15)$ & $0.0332(7)$ \\
\hline C5B & 0.5223 & $0.0742(6)$ & $0.62695(14)$ & $0.0367(7)$ \\
\hline H5B & 0.5863 & 0.1136 & 0.6134 & $0.044^{*}$ \\
\hline C6B & 0.5075 & -0.1315 (6) & $0.66954(15)$ & $0.0371(7)$ \\
\hline C7B & 0.4094 & $-0.1838(7)$ & $0.68957(15)$ & $0.0409(8)$ \\
\hline H7B & 0.3983 & -0.3203 & 0.7183 & $0.049 *$ \\
\hline $\mathrm{C} 8 \mathrm{~B}$ & 0.3303 & $-0.0383(7)$ & $0.66766(15)$ & $0.0415(8)$ \\
\hline H8B & 0.2666 & -0.0768 & 0.6818 & $0.050 *$ \\
\hline C8AB & $0.3444(2)$ & $0.1679(6)$ & $0.62419(15)$ & $0.0373(7)$ \\
\hline N9B & 0.5875 & $-0.2736(7)$ & $0.69421(15)$ & $0.0470(8)$ \\
\hline $\mathrm{H} 3 \mathrm{~A}$ & $0.560(2)$ & $-0.190(7)$ & $0.4877(16)$ & $0.039(10)^{*}$ \\
\hline H3B & $0.383(3)$ & $0.702(7)$ & $0.5125(17)$ & $0.059(12)^{*}$ \\
\hline H9AA & $0.373(3)$ & $0.957(9)$ & 0.2838 (19) & $0.054(12)^{*}$ \\
\hline H9BB & $0.638(3)$ & $-0.297(10)$ & $0.667(2)$ & $0.071(14)^{*}$ \\
\hline H9BA & $0.571(3)$ & $-0.431(9)$ & $0.715(2)$ & $0.062(12)^{*}$ \\
\hline H9AB & $0.307(3)$ & $0.804(8)$ & 0.3255 (19) & $0.050(12)^{*}$ \\
\hline
\end{tabular}

Atomic displacement parameters $\left(\AA^{2}\right)$

\begin{tabular}{lllllll}
\hline & $U^{11}$ & $U^{22}$ & $U^{33}$ & $U^{12}$ & $U^{13}$ & $U^{23}$ \\
\hline O1A & $0.0381(13)$ & $0.0371(12)$ & $0.0429(11)$ & $-0.0020(9)$ & $0.0049(10)$ & $0.0039(10)$ \\
N1A & $0.0368(15)$ & $0.0409(16)$ & $0.0505(18)$ & $-0.0034(13)$ & $0.0035(13)$ & $0.0010(13)$ \\
C2A & $0.0340(18)$ & $0.0394(19)$ & $0.0491(19)$ & $0.0022(14)$ & $0.0003(15)$ & $0.0011(16)$
\end{tabular}




$\begin{array}{lllllll} & & & & \\ \text { N3A } & 0.0399(15) & 0.0334(14) & 0.0385(15) & -0.0004(11) & 0.0010(13) & 0.0035(13) \\ \text { C4A } & 0.0358(17) & 0.0317(15) & 0.0337(16) & -0.0019(12) & 0.0017(13) & -0.0064(13) \\ \text { C4AA } & 0.0376(17) & 0.0307(15) & 0.0332(16) & -0.0035(13) & 0.0013(12) & -0.0058(12) \\ \text { C5A } & 0.0375(17) & 0.0344(16) & 0.0363(17) & -0.0031(13) & 0.0016(14) & -0.0022(14) \\ \text { C6A } & 0.0446(19) & 0.0337(16) & 0.0322(16) & 0.0004(14) & 0.0007(14) & -0.0045(13) \\ \text { C7A } & 0.053(2) & 0.0329(17) & 0.0320(18) & -0.0053(14) & 0.0051(14) & 0.0019(14) \\ \text { C8A } & 0.043(2) & 0.0395(18) & 0.0409(18) & -0.0083(14) & 0.0084(15) & -0.0016(14) \\ \text { C8AA } & 0.0383(17) & 0.0338(17) & 0.0371(17) & -0.0015(14) & 0.0043(14) & -0.0029(14) \\ \text { N9A } & 0.049(2) & 0.0437(17) & 0.0482(18) & 0.0016(14) & -0.0013(15) & 0.0106(15) \\ \text { O1B } & 0.0384(13) & 0.0388(12) & 0.0437(12) & -0.0036(10) & 0.0049(11) & 0.0058(10) \\ \text { N1B } & 0.0365(15) & 0.0463(17) & 0.0525(17) & -0.0017(12) & 0.0019(13) & 0.0057(13) \\ \text { C2B } & 0.0386(19) & 0.0428(18) & 0.0475(19) & 0.0008(14) & -0.0012(15) & 0.0015(16) \\ \text { N3B } & 0.0422(16) & 0.0333(15) & 0.0399(15) & -0.0017(11) & 0.0032(13) & 0.0033(12) \\ \text { C4B } & 0.0393(19) & 0.0291(16) & 0.0313(16) & -0.0029(12) & 0.0016(13) & -0.0014(13) \\ \text { C4AB } & 0.0384(18) & 0.0299(15) & 0.0314(16) & -0.0033(13) & 0.0040(13) & -0.0031(13) \\ \text { C5B } & 0.0389(19) & 0.0350(16) & 0.0361(17) & -0.0041(14) & 0.0047(14) & -0.0041(13) \\ \text { C6B } & 0.0457(19) & 0.0323(16) & 0.0332(17) & -0.0005(14) & -0.0009(14) & -0.0015(14) \\ \text { C7B } & 0.051(2) & 0.0362(18) & 0.0350(17) & -0.0071(15) & 0.0032(15) & 0.0027(14) \\ \text { C8B } & 0.0396(19) & 0.0450(19) & 0.0399(18) & -0.0070(14) & 0.0101(14) & -0.0022(15) \\ \text { C8AB } & 0.0391(17) & 0.0335(16) & 0.0392(17) & -0.0032(13) & 0.0018(14) & -0.0020(14) \\ \text { N9B } & 0.0504(19) & 0.0457(18) & 0.0448(18) & 0.0022(14) & 0.0025(15) & 0.0079(14)\end{array}$

Geometric parameters $\left(A,{ }^{\circ}\right)$

\begin{tabular}{|c|c|c|c|}
\hline $\mathrm{O} 1 \mathrm{~A}-\mathrm{C} 4 \mathrm{~A}$ & $1.235(4)$ & $\mathrm{O} 1 \mathrm{~B}-\mathrm{C} 4 \mathrm{~B}$ & $1.239(4)$ \\
\hline $\mathrm{N} 1 \mathrm{~A}-\mathrm{C} 2 \mathrm{~A}$ & $1.291(5)$ & $\mathrm{N} 1 \mathrm{~B}-\mathrm{C} 2 \mathrm{~B}$ & $1.290(5)$ \\
\hline $\mathrm{N} 1 \mathrm{~A}-\mathrm{C} 8 \mathrm{AA}$ & $1.397(4)$ & $\mathrm{N} 1 \mathrm{~B}-\mathrm{C} 8 \mathrm{AB}$ & $1.391(4)$ \\
\hline $\mathrm{C} 2 \mathrm{~A}-\mathrm{N} 3 \mathrm{~A}$ & $1.369(4)$ & $\mathrm{C} 2 \mathrm{~B}-\mathrm{N} 3 \mathrm{~B}$ & $1.364(4)$ \\
\hline $\mathrm{C} 2 \mathrm{~A}-\mathrm{H} 2 \mathrm{~A}$ & 0.9300 & $\mathrm{C} 2 \mathrm{~B}-\mathrm{H} 2 \mathrm{~B}$ & 0.9300 \\
\hline $\mathrm{N} 3 \mathrm{~A}-\mathrm{C} 4 \mathrm{~A}$ & $1.376(4)$ & $\mathrm{N} 3 \mathrm{~B}-\mathrm{C} 4 \mathrm{~B}$ & $1.366(4)$ \\
\hline $\mathrm{N} 3 \mathrm{~A}-\mathrm{H} 3 \mathrm{~A}$ & $0.94(3)$ & $\mathrm{N} 3 \mathrm{~B}-\mathrm{H} 3 \mathrm{~B}$ & $0.92(2)$ \\
\hline $\mathrm{C} 4 \mathrm{~A}-\mathrm{C} 4 \mathrm{AA}$ & $1.457(4)$ & $\mathrm{C} 4 \mathrm{~B}-\mathrm{C} 4 \mathrm{AB}$ & $1.461(5)$ \\
\hline $\mathrm{C} 4 \mathrm{AA}-\mathrm{C} 5 \mathrm{~A}$ & $1.397(4)$ & $\mathrm{C} 4 \mathrm{AB}-\mathrm{C} 5 \mathrm{~B}$ & $1.393(5)$ \\
\hline $\mathrm{C} 4 \mathrm{AA}-\mathrm{C} 8 \mathrm{AA}$ & $1.405(4)$ & $\mathrm{C} 4 \mathrm{AB}-\mathrm{C} 8 \mathrm{AB}$ & $1.408(4)$ \\
\hline $\mathrm{C} 5 \mathrm{~A}-\mathrm{C} 6 \mathrm{~A}$ & $1.395(5)$ & $\mathrm{C} 5 \mathrm{~B}-\mathrm{C} 6 \mathrm{~B}$ & $1.387(5)$ \\
\hline $\mathrm{C} 5 \mathrm{~A}-\mathrm{H} 5 \mathrm{~A}$ & 0.9300 & $\mathrm{C} 5 \mathrm{~B}-\mathrm{H} 5 \mathrm{~B}$ & 0.9300 \\
\hline $\mathrm{C} 6 \mathrm{~A}-\mathrm{N} 9 \mathrm{~A}$ & $1.374(4)$ & $\mathrm{C} 6 \mathrm{~B}-\mathrm{N} 9 \mathrm{~B}$ & $1.392(5)$ \\
\hline $\mathrm{C} 6 \mathrm{~A}-\mathrm{C} 7 \mathrm{~A}$ & $1.417(5)$ & $\mathrm{C} 6 \mathrm{~B}-\mathrm{C} 7 \mathrm{~B}$ & $1.413(5)$ \\
\hline $\mathrm{C} 7 \mathrm{~A}-\mathrm{C} 8 \mathrm{~A}$ & $1.365(5)$ & $\mathrm{C} 7 \mathrm{~B}-\mathrm{C} 8 \mathrm{~B}$ & $1.370(5)$ \\
\hline $\mathrm{C} 7 \mathrm{~A}-\mathrm{H} 7 \mathrm{~A}$ & 0.9300 & $\mathrm{C} 7 \mathrm{~B}-\mathrm{H} 7 \mathrm{~B}$ & 0.9300 \\
\hline $\mathrm{C} 8 \mathrm{~A}-\mathrm{C} 8 \mathrm{AA}$ & $1.403(5)$ & $\mathrm{C} 8 \mathrm{~B}-\mathrm{C} 8 \mathrm{AB}$ & $1.400(5)$ \\
\hline $\mathrm{C} 8 \mathrm{~A}-\mathrm{H} 8 \mathrm{~A}$ & 0.9300 & $\mathrm{C} 8 \mathrm{~B}-\mathrm{H} 8 \mathrm{~B}$ & 0.9300 \\
\hline N9A-H9AA & $0.93(4)$ & N9B-H9BB & $0.90(5)$ \\
\hline $\mathrm{N} 9 \mathrm{~A}-\mathrm{H} 9 \mathrm{AB}$ & $0.81(4)$ & N9B-H9BA & $0.93(5)$ \\
\hline $\mathrm{C} 2 \mathrm{~A}-\mathrm{N} 1 \mathrm{~A}-\mathrm{C} 8 \mathrm{AA}$ & $116.3(3)$ & $\mathrm{C} 2 \mathrm{~B}-\mathrm{N} 1 \mathrm{~B}-\mathrm{C} 8 \mathrm{AB}$ & $116.6(3)$ \\
\hline $\mathrm{N} 1 \mathrm{~A}-\mathrm{C} 2 \mathrm{~A}-\mathrm{N} 3 \mathrm{~A}$ & $124.8(3)$ & $\mathrm{N} 1 \mathrm{~B}-\mathrm{C} 2 \mathrm{~B}-\mathrm{N} 3 \mathrm{~B}$ & $124.9(3)$ \\
\hline $\mathrm{N} 1 \mathrm{~A}-\mathrm{C} 2 \mathrm{~A}-\mathrm{H} 2 \mathrm{~A}$ & 117.6 & $\mathrm{~N} 1 \mathrm{~B}-\mathrm{C} 2 \mathrm{~B}-\mathrm{H} 2 \mathrm{~B}$ & 117.6 \\
\hline
\end{tabular}




\begin{tabular}{|c|c|}
\hline $\mathrm{N} 3 \mathrm{~A}-\mathrm{C} 2 \mathrm{~A}-\mathrm{H} 2 \mathrm{~A}$ & 117.6 \\
\hline $\mathrm{C} 2 \mathrm{~A}-\mathrm{N} 3 \mathrm{~A}-\mathrm{C} 4 \mathrm{~A}$ & $123.2(3)$ \\
\hline $\mathrm{C} 2 \mathrm{~A}-\mathrm{N} 3 \mathrm{~A}-\mathrm{H} 3 \mathrm{~A}$ & $120(2)$ \\
\hline $\mathrm{C} 4 \mathrm{~A}-\mathrm{N} 3 \mathrm{~A}-\mathrm{H} 3 \mathrm{~A}$ & $117(2)$ \\
\hline $\mathrm{O} 1 \mathrm{~A}-\mathrm{C} 4 \mathrm{~A}-\mathrm{N} 3 \mathrm{~A}$ & $120.9(3)$ \\
\hline $\mathrm{O} 1 \mathrm{~A}-\mathrm{C} 4 \mathrm{~A}-\mathrm{C} 4 \mathrm{AA}$ & $124.9(3)$ \\
\hline $\mathrm{N} 3 \mathrm{~A}-\mathrm{C} 4 \mathrm{~A}-\mathrm{C} 4 \mathrm{AA}$ & $114.3(3)$ \\
\hline $\mathrm{C} 5 \mathrm{~A}-\mathrm{C} 4 \mathrm{AA}-\mathrm{C} 8 \mathrm{AA}$ & $120.8(3)$ \\
\hline $\mathrm{C} 5 \mathrm{~A}-\mathrm{C} 4 \mathrm{AA}-\mathrm{C} 4 \mathrm{~A}$ & $120.6(3)$ \\
\hline $\mathrm{C} 8 \mathrm{AA}-\mathrm{C} 4 \mathrm{AA}-\mathrm{C} 4 \mathrm{~A}$ & $118.6(3)$ \\
\hline $\mathrm{C} 6 \mathrm{~A}-\mathrm{C} 5 \mathrm{~A}-\mathrm{C} 4 \mathrm{AA}$ & $120.7(3)$ \\
\hline $\mathrm{C} 6 \mathrm{~A}-\mathrm{C} 5 \mathrm{~A}-\mathrm{H} 5 \mathrm{~A}$ & 119.6 \\
\hline $\mathrm{C} 4 \mathrm{AA}-\mathrm{C} 5 \mathrm{~A}-\mathrm{H} 5 \mathrm{~A}$ & 119.6 \\
\hline $\mathrm{N} 9 \mathrm{~A}-\mathrm{C} 6 \mathrm{~A}-\mathrm{C} 5 \mathrm{~A}$ & $121.7(3)$ \\
\hline $\mathrm{N} 9 \mathrm{~A}-\mathrm{C} 6 \mathrm{~A}-\mathrm{C} 7 \mathrm{~A}$ & $120.4(3)$ \\
\hline $\mathrm{C} 5 \mathrm{~A}-\mathrm{C} 6 \mathrm{~A}-\mathrm{C} 7 \mathrm{~A}$ & $117.8(3)$ \\
\hline $\mathrm{C} 8 \mathrm{~A}-\mathrm{C} 7 \mathrm{~A}-\mathrm{C} 6 \mathrm{~A}$ & $121.5(3)$ \\
\hline $\mathrm{C} 8 \mathrm{~A}-\mathrm{C} 7 \mathrm{~A}-\mathrm{H} 7 \mathrm{~A}$ & 119.3 \\
\hline $\mathrm{C} 6 \mathrm{~A}-\mathrm{C} 7 \mathrm{~A}-\mathrm{H} 7 \mathrm{~A}$ & 119.3 \\
\hline $\mathrm{C} 7 \mathrm{~A}-\mathrm{C} 8 \mathrm{~A}-\mathrm{C} 8 \mathrm{AA}$ & $121.0(3)$ \\
\hline $\mathrm{C} 7 \mathrm{~A}-\mathrm{C} 8 \mathrm{~A}-\mathrm{H} 8 \mathrm{~A}$ & 119.5 \\
\hline $\mathrm{C} 8 \mathrm{AA}-\mathrm{C} 8 \mathrm{~A}-\mathrm{H} 8 \mathrm{~A}$ & 119.5 \\
\hline $\mathrm{N} 1 \mathrm{~A}-\mathrm{C} 8 \mathrm{AA}-\mathrm{C} 8 \mathrm{~A}$ & $119.0(3)$ \\
\hline $\mathrm{N} 1 \mathrm{~A}-\mathrm{C} 8 \mathrm{AA}-\mathrm{C} 4 \mathrm{AA}$ & $122.8(3)$ \\
\hline $\mathrm{C} 8 \mathrm{~A}-\mathrm{C} 8 \mathrm{AA}-\mathrm{C} 4 \mathrm{AA}$ & $118.2(3)$ \\
\hline $\mathrm{C} 6 \mathrm{~A}-\mathrm{N} 9 \mathrm{~A}-\mathrm{H} 9 \mathrm{AA}$ & $117(3)$ \\
\hline $\mathrm{C} 6 \mathrm{~A}-\mathrm{N} 9 \mathrm{~A}-\mathrm{H} 9 \mathrm{AB}$ & $116(3)$ \\
\hline H9AA-N9A-H9AB & $116(4)$ \\
\hline $\mathrm{C} 8 \mathrm{AA}-\mathrm{N} 1 \mathrm{~A}-\mathrm{C} 2 \mathrm{~A}-\mathrm{N} 3 \mathrm{~A}$ & $-0.4(5)$ \\
\hline $\mathrm{N} 1 \mathrm{~A}-\mathrm{C} 2 \mathrm{~A}-\mathrm{N} 3 \mathrm{~A}-\mathrm{C} 4 \mathrm{~A}$ & $0.2(5)$ \\
\hline $\mathrm{C} 2 \mathrm{~A}-\mathrm{N} 3 \mathrm{~A}-\mathrm{C} 4 \mathrm{~A}-\mathrm{O} 1 \mathrm{~A}$ & $-179.7(3)$ \\
\hline $\mathrm{C} 2 \mathrm{~A}-\mathrm{N} 3 \mathrm{~A}-\mathrm{C} 4 \mathrm{~A}-\mathrm{C} 4 \mathrm{AA}$ & $-0.1(4)$ \\
\hline $\mathrm{O} 1 \mathrm{~A}-\mathrm{C} 4 \mathrm{~A}-\mathrm{C} 4 \mathrm{AA}-\mathrm{C} 5 \mathrm{~A}$ & $-0.5(5)$ \\
\hline $\mathrm{N} 3 \mathrm{~A}-\mathrm{C} 4 \mathrm{~A}-\mathrm{C} 4 \mathrm{AA}-\mathrm{C} 5 \mathrm{~A}$ & $179.9(3)$ \\
\hline $\mathrm{O} 1 \mathrm{~A}-\mathrm{C} 4 \mathrm{~A}-\mathrm{C} 4 \mathrm{AA}-\mathrm{C} 8 \mathrm{AA}$ & $179.8(3)$ \\
\hline $\mathrm{N} 3 \mathrm{~A}-\mathrm{C} 4 \mathrm{~A}-\mathrm{C} 4 \mathrm{AA}-\mathrm{C} 8 \mathrm{AA}$ & $0.2(4)$ \\
\hline $\mathrm{C} 8 \mathrm{AA}-\mathrm{C} 4 \mathrm{AA}-\mathrm{C} 5 \mathrm{~A}-\mathrm{C} 6 \mathrm{~A}$ & $0.1(5)$ \\
\hline $\mathrm{C} 4 \mathrm{~A}-\mathrm{C} 4 \mathrm{AA}-\mathrm{C} 5 \mathrm{~A}-\mathrm{C} 6 \mathrm{~A}$ & $-179.6(3)$ \\
\hline $\mathrm{C} 4 \mathrm{AA}-\mathrm{C} 5 \mathrm{~A}-\mathrm{C} 6 \mathrm{~A}-\mathrm{N} 9 \mathrm{~A}$ & $177.6(3)$ \\
\hline $\mathrm{C} 4 \mathrm{AA}-\mathrm{C} 5 \mathrm{~A}-\mathrm{C} 6 \mathrm{~A}-\mathrm{C} 7 \mathrm{~A}$ & $-0.1(5)$ \\
\hline $\mathrm{N} 9 \mathrm{~A}-\mathrm{C} 6 \mathrm{~A}-\mathrm{C} 7 \mathrm{~A}-\mathrm{C} 8 \mathrm{~A}$ & $-177.4(3)$ \\
\hline $\mathrm{C} 5 \mathrm{~A}-\mathrm{C} 6 \mathrm{~A}-\mathrm{C} 7 \mathrm{~A}-\mathrm{C} 8 \mathrm{~A}$ & $0.4(5)$ \\
\hline $\mathrm{C} 6 \mathrm{~A}-\mathrm{C} 7 \mathrm{~A}-\mathrm{C} 8 \mathrm{~A}-\mathrm{C} 8 \mathrm{AA}$ & $-0.6(5)$ \\
\hline $\mathrm{C} 2 \mathrm{~A}-\mathrm{N} 1 \mathrm{~A}-\mathrm{C} 8 \mathrm{AA}-\mathrm{C} 8 \mathrm{~A}$ & $-179.3(3)$ \\
\hline $\mathrm{C} 2 \mathrm{~A}-\mathrm{N} 1 \mathrm{~A}-\mathrm{C} 8 \mathrm{AA}-\mathrm{C} 4 \mathrm{AA}$ & $0.6(5)$ \\
\hline $\mathrm{C} 7 \mathrm{~A}-\mathrm{C} 8 \mathrm{~A}-\mathrm{C} 8 \mathrm{AA}-\mathrm{N} 1 \mathrm{~A}$ & $-179.6(3)$ \\
\hline $\mathrm{C} 7 \mathrm{~A}-\mathrm{C} 8 \mathrm{~A}-\mathrm{C} 8 \mathrm{AA}-\mathrm{C} 4 \mathrm{AA}$ & $0.5(5)$ \\
\hline
\end{tabular}

$\mathrm{N} 3 \mathrm{~B}-\mathrm{C} 2 \mathrm{~B}-\mathrm{H} 2 \mathrm{~B} \quad 117.6$

$\mathrm{C} 2 \mathrm{~B}-\mathrm{N} 3 \mathrm{~B}-\mathrm{C} 4 \mathrm{~B} \quad 123.0(3)$

$\mathrm{C} 2 \mathrm{~B}-\mathrm{N} 3 \mathrm{~B}-\mathrm{H} 3 \mathrm{~B} \quad 123(2)$

$\mathrm{C} 4 \mathrm{~B}-\mathrm{N} 3 \mathrm{~B}-\mathrm{H} 3 \mathrm{~B} \quad 114(2)$

$\mathrm{O} 1 \mathrm{~B}-\mathrm{C} 4 \mathrm{~B}-\mathrm{N} 3 \mathrm{~B} \quad 121.3(3)$

$\mathrm{O} 1 \mathrm{~B}-\mathrm{C} 4 \mathrm{~B}-\mathrm{C} 4 \mathrm{AB} \quad 123.9(3)$

$\mathrm{N} 3 \mathrm{~B}-\mathrm{C} 4 \mathrm{~B}-\mathrm{C} 4 \mathrm{AB} \quad 114.7$ (3)

$\mathrm{C} 5 \mathrm{~B}-\mathrm{C} 4 \mathrm{AB}-\mathrm{C} 8 \mathrm{AB} \quad 121.0(3)$

$\mathrm{C} 5 \mathrm{~B}-\mathrm{C} 4 \mathrm{AB}-\mathrm{C} 4 \mathrm{~B} \quad 120.9(3)$

$\mathrm{C} 8 \mathrm{AB}-\mathrm{C} 4 \mathrm{AB}-\mathrm{C} 4 \mathrm{~B} \quad 118.1(3)$

$\mathrm{C} 6 \mathrm{~B}-\mathrm{C} 5 \mathrm{~B}-\mathrm{C} 4 \mathrm{AB} \quad 120.6(3)$

$\mathrm{C} 6 \mathrm{~B}-\mathrm{C} 5 \mathrm{~B}-\mathrm{H} 5 \mathrm{~B} \quad 119.7$

$\mathrm{C} 4 \mathrm{AB}-\mathrm{C} 5 \mathrm{~B}-\mathrm{H} 5 \mathrm{~B} \quad 119.7$

$\mathrm{C} 5 \mathrm{~B}-\mathrm{C} 6 \mathrm{~B}-\mathrm{N} 9 \mathrm{~B} \quad 121.0(3)$

$\mathrm{C} 5 \mathrm{~B}-\mathrm{C} 6 \mathrm{~B}-\mathrm{C} 7 \mathrm{~B} \quad 118.1$ (3)

$\mathrm{N} 9 \mathrm{~B}-\mathrm{C} 6 \mathrm{~B}-\mathrm{C} 7 \mathrm{~B} \quad 120.8(3)$

$\mathrm{C} 8 \mathrm{~B}-\mathrm{C} 7 \mathrm{~B}-\mathrm{C} 6 \mathrm{~B} \quad 121.6(3)$

$\mathrm{C} 8 \mathrm{~B}-\mathrm{C} 7 \mathrm{~B}-\mathrm{H} 7 \mathrm{~B} \quad 119.2$

$\mathrm{C} 6 \mathrm{~B}-\mathrm{C} 7 \mathrm{~B}-\mathrm{H} 7 \mathrm{~B} \quad 119.2$

$\mathrm{C} 7 \mathrm{~B}-\mathrm{C} 8 \mathrm{~B}-\mathrm{C} 8 \mathrm{AB} \quad 120.7$ (3)

$\mathrm{C} 7 \mathrm{~B}-\mathrm{C} 8 \mathrm{~B}-\mathrm{H} 8 \mathrm{~B} \quad 119.7$

$\mathrm{C} 8 \mathrm{AB}-\mathrm{C} 8 \mathrm{~B}-\mathrm{H} 8 \mathrm{~B} \quad 119.7$

N1B-C8AB-C8B 119.4 (3)

$\mathrm{N} 1 \mathrm{~B}-\mathrm{C} 8 \mathrm{AB}-\mathrm{C} 4 \mathrm{AB} \quad 122.6(3)$

$\mathrm{C} 8 \mathrm{~B}-\mathrm{C} 8 \mathrm{AB}-\mathrm{C} 4 \mathrm{AB} \quad 118.0$ (3)

$\mathrm{C} 6 \mathrm{~B}-\mathrm{N} 9 \mathrm{~B}-\mathrm{H} 9 \mathrm{BB} \quad 113(3)$

C6B-N9B-H9BA 116 (3)

H9BB-N9B-H9BA 113 (4)

$\mathrm{C} 8 \mathrm{AB}-\mathrm{N} 1 \mathrm{~B}-\mathrm{C} 2 \mathrm{~B}-\mathrm{N} 3 \mathrm{~B} \quad-0.9(5)$

$\mathrm{N} 1 \mathrm{~B}-\mathrm{C} 2 \mathrm{~B}-\mathrm{N} 3 \mathrm{~B}-\mathrm{C} 4 \mathrm{~B} \quad 1.6(5)$

$\mathrm{C} 2 \mathrm{~B}-\mathrm{N} 3 \mathrm{~B}-\mathrm{C} 4 \mathrm{~B}-\mathrm{O} 1 \mathrm{~B} \quad 178.9$ (3)

$\mathrm{C} 2 \mathrm{~B}-\mathrm{N} 3 \mathrm{~B}-\mathrm{C} 4 \mathrm{~B}-\mathrm{C} 4 \mathrm{AB} \quad-0.8(4)$

$\mathrm{O} 1 \mathrm{~B}-\mathrm{C} 4 \mathrm{~B}-\mathrm{C} 4 \mathrm{AB}-\mathrm{C} 5 \mathrm{~B} \quad-0.2(5)$

$\mathrm{N} 3 \mathrm{~B}-\mathrm{C} 4 \mathrm{~B}-\mathrm{C} 4 \mathrm{AB}-\mathrm{C} 5 \mathrm{~B} \quad 179.5(3)$

$\mathrm{O} 1 \mathrm{~B}-\mathrm{C} 4 \mathrm{~B}-\mathrm{C} 4 \mathrm{AB}-\mathrm{C} 8 \mathrm{AB} \quad 179.8(3)$

$\mathrm{N} 3 \mathrm{~B}-\mathrm{C} 4 \mathrm{~B}-\mathrm{C} 4 \mathrm{AB}-\mathrm{C} 8 \mathrm{AB} \quad-0.5$ (4)

$\mathrm{C} 8 \mathrm{AB}-\mathrm{C} 4 \mathrm{AB}-\mathrm{C} 5 \mathrm{~B}-\mathrm{C} 6 \mathrm{~B} \quad 0.2(5)$

$\mathrm{C} 4 \mathrm{~B}-\mathrm{C} 4 \mathrm{AB}-\mathrm{C} 5 \mathrm{~B}-\mathrm{C} 6 \mathrm{~B}-179.8(3)$

$\mathrm{C} 4 \mathrm{AB}-\mathrm{C} 5 \mathrm{~B}-\mathrm{C} 6 \mathrm{~B}-\mathrm{N} 9 \mathrm{~B} \quad-177.5(3)$

$\mathrm{C} 4 \mathrm{AB}-\mathrm{C} 5 \mathrm{~B}-\mathrm{C} 6 \mathrm{~B}-\mathrm{C} 7 \mathrm{~B} \quad-0.6(5)$

$\mathrm{C} 5 \mathrm{~B}-\mathrm{C} 6 \mathrm{~B}-\mathrm{C} 7 \mathrm{~B}-\mathrm{C} 8 \mathrm{~B} \quad 0.4(5)$

$\mathrm{N} 9 \mathrm{~B}-\mathrm{C} 6 \mathrm{~B}-\mathrm{C} 7 \mathrm{~B}-\mathrm{C} 8 \mathrm{~B} \quad 177.3$ (3)

$\mathrm{C} 6 \mathrm{~B}-\mathrm{C} 7 \mathrm{~B}-\mathrm{C} 8 \mathrm{~B}-\mathrm{C} 8 \mathrm{AB} \quad 0.2(5)$

$\mathrm{C} 2 \mathrm{~B}-\mathrm{N} 1 \mathrm{~B}-\mathrm{C} 8 \mathrm{AB}-\mathrm{C} 8 \mathrm{~B} \quad-179.7$ (3)

$\mathrm{C} 2 \mathrm{~B}-\mathrm{N} 1 \mathrm{~B}-\mathrm{C} 8 \mathrm{AB}-\mathrm{C} 4 \mathrm{AB} \quad-0.5(5)$

$\mathrm{C} 7 \mathrm{~B}-\mathrm{C} 8 \mathrm{~B}-\mathrm{C} 8 \mathrm{AB}-\mathrm{N} 1 \mathrm{~B} \quad 178.6(3)$

$\mathrm{C} 7 \mathrm{~B}-\mathrm{C} 8 \mathrm{~B}-\mathrm{C} 8 \mathrm{AB}-\mathrm{C} 4 \mathrm{AB} \quad-0.6(5)$ 


$\begin{array}{llll}\mathrm{C} 5 \mathrm{~A}-\mathrm{C} 4 \mathrm{AA}-\mathrm{C} 8 \mathrm{AA}-\mathrm{N} 1 \mathrm{~A} & 179.8(3) & \mathrm{C} 5 \mathrm{~B}-\mathrm{C} 4 \mathrm{AB}-\mathrm{C} 8 \mathrm{AB}-\mathrm{N} 1 \mathrm{~B} & -178.8(3) \\ \mathrm{C} 4 \mathrm{~A}-\mathrm{C} 4 \mathrm{AA}-\mathrm{C} 8 \mathrm{AA}-\mathrm{N} 1 \mathrm{~A} & -0.5(4) & \mathrm{C} 4 \mathrm{~B}-\mathrm{C} 4 \mathrm{AB}-\mathrm{C} 8 \mathrm{AB}-\mathrm{N} 1 \mathrm{~B} & 1.2(5) \\ \mathrm{C} 5 \mathrm{~A}-\mathrm{C} 4 \mathrm{AA}-\mathrm{C} 8 \mathrm{AA}-\mathrm{C} 8 \mathrm{~A} & -0.3(4) & \mathrm{C} 5 \mathrm{~B}-\mathrm{C} 4 \mathrm{AB}-\mathrm{C} 8 \mathrm{AB}-\mathrm{C} 8 \mathrm{~B} & 0.4(4) \\ \mathrm{C} 4 \mathrm{~A}-\mathrm{C} 4 \mathrm{AA}-\mathrm{C} 8 \mathrm{AA}-\mathrm{C} 8 \mathrm{~A} & 179.4(3) & \mathrm{C} 4 \mathrm{~B}-\mathrm{C} 4 \mathrm{AB}-\mathrm{C} 8 \mathrm{AB}-\mathrm{C} 8 \mathrm{~B} & -179.6(3)\end{array}$

Hydrogen-bond geometry $\left(A,{ }^{\circ}\right)$

\begin{tabular}{lllll}
\hline$D-\mathrm{H} \cdots A$ & $D-\mathrm{H}$ & $\mathrm{H} \cdots A$ & $D \cdots A$ & $D-\mathrm{H} \cdots A$ \\
\hline $\mathrm{N} 9 A-\mathrm{H} 9 A A \cdots \mathrm{N} 9 B^{\mathrm{i}}$ & $0.93(4)$ & $2.55(4)$ & $3.435(5)$ & $160(4)$ \\
$\mathrm{N} 9 A-\mathrm{H} 9 A B \cdots \mathrm{N} 1 A^{\mathrm{ii}}$ & $0.81(4)$ & $2.34(4)$ & $3.144(5)$ & $170(4)$ \\
$\mathrm{N} 9 B-\mathrm{H} 9 B B \cdots \mathrm{N} 1 B^{\mathrm{iii}}$ & $0.91(4)$ & $2.19(4)$ & $3.092(5)$ & $174(4)$ \\
$\mathrm{N} 3 A-\mathrm{H} 3 A \cdots \mathrm{O} 1 B^{\mathrm{i}}$ & $0.95(3)$ & $1.89(3)$ & $2.832(4)$ & $175(3)$ \\
$\mathrm{N} 3 B-\mathrm{H} 3 B \cdots \mathrm{O} 1 A^{\mathrm{v}}$ & $0.92(4)$ & $1.93(4)$ & $2.847(3)$ & $173(4)$ \\
\hline
\end{tabular}

Symmetry codes: (i) $-x+1,-y+1, z-1 / 2$; (ii) $x-1 / 2,-y+1, z$; (iii) $x+1 / 2,-y, z$; (iv) $x, y-1, z$; (v) $x, y+1, z$.

4-Aminoquinazolin-1-ium chloride-4-aminoquinazoline-water (1/1/2) (III)

Crystal data

$\mathrm{C}_{8} \mathrm{H}_{8} \mathrm{~N}_{3}{ }^{+} \cdot \mathrm{Cl}^{-} \cdot \mathrm{C}_{8} \mathrm{H}_{7} \mathrm{~N}_{3} \cdot 2 \mathrm{H}_{2} \mathrm{O}$

$M_{r}=362.82$

Monoclinic, $P 2{ }_{1} / n$

$a=14.3512(12) \AA$

$b=7.5867(6) \AA$

$c=16.2282(9) \AA$

$\beta=93.544(7)^{\circ}$

$V=1763.5(2) \AA^{3}$

$Z=4$

\section{Data collection}

Rigaku Xcalibur, Ruby diffractometer

Radiation source: Enhance (Cu) X-ray Source Graphite monochromator

Detector resolution: 10.2576 pixels $\mathrm{mm}^{-1}$

$\omega$ scans

Absorption correction: multi-scan

(CrysAlisPro; Rigaku OD, 2018)

$T_{\min }=0.934, T_{\max }=1.000$

\section{Refinement}

Refinement on $F^{2}$

Least-squares matrix: full

$R\left[F^{2}>2 \sigma\left(F^{2}\right)\right]=0.054$

$w R\left(F^{2}\right)=0.151$

$S=1.00$

3563 reflections

261 parameters

4 restraints
$F(000)=760$

$D_{\mathrm{x}}=1.367 \mathrm{Mg} \mathrm{m}^{-3}$

$\mathrm{Cu} K \alpha$ radiation, $\lambda=1.54184 \AA$

Cell parameters from 1071 reflections

$\theta=4.0-71.2^{\circ}$

$\mu=2.12 \mathrm{~mm}^{-1}$

$T=298 \mathrm{~K}$

Needle, colourless

$0.50 \times 0.08 \times 0.05 \mathrm{~mm}$

6703 measured reflections

3563 independent reflections

2207 reflections with $I>2 \sigma(I)$

$R_{\text {int }}=0.052$

$\theta_{\max }=75.8^{\circ}, \theta_{\min }=4.0^{\circ}$

$h=-17 \rightarrow 15$

$k=-9 \rightarrow 9$

$l=-15 \rightarrow 19$

Primary atom site location: structure-invariant direct methods

Hydrogen site location: mixed

$\mathrm{H}$ atoms treated by a mixture of independent and constrained refinement

$w=1 /\left[\sigma^{2}\left(F_{\mathrm{o}}^{2}\right)+(0.0514 P)^{2}\right]$

where $P=\left(F_{\mathrm{o}}^{2}+2 F_{\mathrm{c}}{ }^{2}\right) / 3$

$(\Delta / \sigma)_{\max }=0.003$

$\Delta \rho_{\max }=0.23 \mathrm{e} \AA^{-3}$

$\Delta \rho_{\min }=-0.21$ e $\AA^{-3}$ 


\section{Special details}

Geometry. All esds (except the esd in the dihedral angle between two 1.s. planes) are estimated using the full covariance matrix. The cell esds are taken into account individually in the estimation of esds in distances, angles and torsion angles; correlations between esds in cell parameters are only used when they are defined by crystal symmetry. An approximate (isotropic) treatment of cell esds is used for estimating esds involving l.s. planes.

Fractional atomic coordinates and isotropic or equivalent isotropic displacement parameters $\left(\AA^{2}\right)$

\begin{tabular}{|c|c|c|c|c|}
\hline & $x$ & $y$ & $z$ & $U_{\text {iso }} * / U_{\text {eq }}$ \\
\hline $\mathrm{Cl1}$ & $0.38300(8)$ & $0.88404(15)$ & $0.76044(5)$ & 0.0719 \\
\hline $\mathrm{O} 1 \mathrm{~W}$ & $0.3293(2)$ & $0.4885(5)$ & $0.72013(16)$ & $0.0732(8)$ \\
\hline $\mathrm{O} 2 \mathrm{~W}$ & 0.4789 (2) & $0.2659(6)$ & $0.7806(2)$ & $0.0896(10)$ \\
\hline N1A & $0.28852(17)$ & $0.9176(4)$ & $0.34296(12)$ & $0.0423(6)$ \\
\hline $\mathrm{C} 2 \mathrm{~A}$ & $0.3708(2)$ & 0.8430 & $0.35562(16)$ & $0.0440(7)$ \\
\hline $\mathrm{H} 2 \mathrm{AA}$ & 0.4007 & 0.8052 & 0.3095 & $0.053^{*}$ \\
\hline $\mathrm{N} 3 \mathrm{~A}$ & $0.41460(17)$ & $0.8174(4)$ & $0.42915(13)$ & 0.0408 \\
\hline $\mathrm{C} 4 \mathrm{~A}$ & $0.3713(2)$ & $0.8717(4)$ & $0.49712(16)$ & $0.0396(6)$ \\
\hline C4AA & $0.28014(19)$ & $0.9559(4)$ & $0.48907(16)$ & $0.0389(6)$ \\
\hline C5A & $0.2313(2)$ & $1.0135(5)$ & $0.55595(17)$ & $0.0484(7)$ \\
\hline H5AA & 0.2578 & 1.0010 & 0.6094 & $0.058^{*}$ \\
\hline C6A & $0.1456(2)$ & $1.0874(5)$ & $0.5433(2)$ & $0.0551(8)$ \\
\hline H6AA & 0.1133 & 1.1248 & 0.5881 & $0.066^{*}$ \\
\hline C7A & $0.1056(2)$ & $1.1074(5)$ & $0.4628(2)$ & 0.0527 \\
\hline H7AA & 0.0468 & 1.1584 & 0.4546 & $0.063 *$ \\
\hline C8A & $0.1520(2)$ & $1.0528(4)$ & 0.39587 (17) & $0.0469(7)$ \\
\hline H8AA & 0.1253 & 1.0664 & 0.3426 & $0.056^{*}$ \\
\hline C8AA & $0.23995(19)$ & $0.9765(4)$ & $0.40943(16)$ & $0.0385(6)$ \\
\hline N9A & 0.41535 (19) & $0.8422(4)$ & $0.56770(14)$ & $0.0505(7)$ \\
\hline N1B & $0.28423(18)$ & $0.4265(4)$ & $0.32016(12)$ & $0.0435(6)$ \\
\hline $\mathrm{C} 2 \mathrm{~B}$ & $0.3657(2)$ & $0.3578(4)$ & $0.34201(16)$ & $0.0447(7)$ \\
\hline H2BA & 0.4010 & 0.3203 & 0.2991 & $0.054^{*}$ \\
\hline N3B & $0.40470(17)$ & $0.3348(4)$ & $0.41844(14)$ & $0.0431(6)$ \\
\hline $\mathrm{C} 4 \mathrm{~B}$ & $0.35638(19)$ & $0.3916(4)$ & $0.48186(15)$ & $0.0382(6)$ \\
\hline $\mathrm{C} 4 \mathrm{AB}$ & $0.26521(19)$ & 0.4695 (4) & $0.46662(15)$ & $0.0359(5)$ \\
\hline $\mathrm{C} 5 \mathrm{~B}$ & $0.2086(2)$ & $0.5269(4)$ & $0.52924(16)$ & $0.0426(6)$ \\
\hline H5BA & 0.2304 & 0.5181 & 0.5843 & $0.051 *$ \\
\hline C6B & $0.1221(2)$ & $0.5954(4)$ & $0.50991(19)$ & $0.0486(7)$ \\
\hline H6BA & 0.0856 & 0.6345 & 0.5516 & $0.058^{*}$ \\
\hline C7B & 0.0883 & $0.6069(4)$ & $0.42737(19)$ & $0.0481(7)$ \\
\hline H7BA & 0.0290 & 0.6522 & 0.4146 & $0.058 *$ \\
\hline C8B & $0.1419(2)$ & $0.5522(4)$ & $0.36512(16)$ & $0.0439(7)$ \\
\hline H8BA & 0.1189 & 0.5615 & 0.3104 & $0.053^{*}$ \\
\hline C8AB & $0.23112(19)$ & 0.4821 & $0.38354(15)$ & $0.0383(6)$ \\
\hline N9B & $0.39542(19)$ & $0.3709(4)$ & $0.55724(14)$ & $0.0493(7)$ \\
\hline $\mathrm{H} 1 \mathrm{~A}$ & $0.259(3)$ & $0.929(5)$ & $0.284(2)$ & $0.060(10)^{*}$ \\
\hline H9AA & $0.472(3)$ & $0.789(5)$ & $0.569(2)$ & $0.062(11)^{*}$ \\
\hline H9AB & $0.390(4)$ & $0.880(7)$ & $0.617(3)$ & $0.103(17)^{*}$ \\
\hline H9BA & $0.453(3)$ & $0.308(5)$ & $0.565(2)$ & $0.057(10)^{*}$ \\
\hline
\end{tabular}




\begin{tabular}{|c|c|c|c|c|}
\hline H9BB & $0.370(3)$ & $0.406(5)$ & $0.596(2)$ & $0.047(9)^{*}$ \\
\hline H1W1 & $0.345(4)$ & $0.601(4)$ & $0.733(3)$ & $0.11(2)^{*}$ \\
\hline $\mathrm{H} 2 \mathrm{~W} 1$ & $0.271(2)$ & $0.497(11)$ & $0.736(4)$ & $0.19(4)^{*}$ \\
\hline H1W2 & $0.437(5)$ & $0.346(8)$ & $0.759(5)$ & $0.190^{*}$ \\
\hline $\mathrm{H} 2 \mathrm{~W} 2$ & $0.437(4)$ & $0.183(7)$ & $0.766(4)$ & $0.15(3) *$ \\
\hline
\end{tabular}

Atomic displacement parameters $\left(\AA^{2}\right)$

\begin{tabular}{lllllll}
\hline & $U^{11}$ & $U^{22}$ & $U^{33}$ & $U^{12}$ & $U^{13}$ & $U^{23}$ \\
\hline Cl1 & $0.0881(7)$ & $0.0841(7)$ & $0.0431(4)$ & $-0.0031(6)$ & $0.0015(4)$ & $-0.0039(4)$ \\
O1W & $0.082(2)$ & $0.081(2)$ & $0.0566(13)$ & $0.0059(17)$ & $0.0021(13)$ & $-0.0063(14)$ \\
O2W & $0.0612(18)$ & $0.097(3)$ & $0.109(2)$ & $0.0033(19)$ & $-0.0051(17)$ & $-0.011(2)$ \\
N1A & $0.0404(13)$ & $0.0530(16)$ & $0.0329(10)$ & $-0.0007(11)$ & $-0.0021(9)$ & $0.0007(10)$ \\
C2A & $0.0400(15)$ & $0.0517(18)$ & $0.0409(12)$ & $-0.0035(13)$ & $0.0075(11)$ & $-0.0031(12)$ \\
N3A & $0.0333(11)$ & $0.0498(15)$ & $0.0396(10)$ & $0.0055(10)$ & $0.0051(9)$ & $0.0006(10)$ \\
C4A & $0.0392(14)$ & $0.0415(15)$ & $0.0382(12)$ & $-0.0001(12)$ & $0.0038(10)$ & $-0.0021(11)$ \\
C4AA & $0.0346(14)$ & $0.0380(15)$ & $0.0439(13)$ & $0.0001(12)$ & $0.0013(10)$ & $0.0021(11)$ \\
C5A & $0.0524(18)$ & $0.0528(19)$ & $0.0399(13)$ & $0.0034(15)$ & $0.0030(12)$ & $0.0010(12)$ \\
C6A & $0.0546(19)$ & $0.055(2)$ & $0.0569(16)$ & $0.0085(16)$ & $0.0165(14)$ & $-0.0047(15)$ \\
C7A & $0.0345(15)$ & $0.0489(18)$ & $0.0751(19)$ & $0.0105(14)$ & $0.0058(13)$ & $0.0000(16)$ \\
C8A & $0.0469(17)$ & $0.0473(18)$ & $0.0453(13)$ & $-0.0030(14)$ & $-0.0075(12)$ & $0.0040(12)$ \\
C8AA & $0.0377(14)$ & $0.0373(14)$ & $0.0409(12)$ & $-0.0032(12)$ & $0.0068(10)$ & $0.0018(11)$ \\
N9A & $0.0406(14)$ & $0.072(2)$ & $0.0390(11)$ & $0.0133(13)$ & $0.0009(10)$ & $-0.0045(11)$ \\
N1B & $0.0443(13)$ & $0.0537(16)$ & $0.0320(9)$ & $0.0025(12)$ & $-0.0013(9)$ & $-0.0011(10)$ \\
C2B & $0.0423(15)$ & $0.0527(18)$ & $0.0397(12)$ & $0.0029(13)$ & $0.0060(11)$ & $-0.0032(12)$ \\
N3B & $0.0338(12)$ & $0.0563(16)$ & $0.0391(10)$ & $0.0066(11)$ & $0.0010(9)$ & $-0.0042(10)$ \\
C4B & $0.0340(13)$ & $0.0436(15)$ & $0.0368(11)$ & $-0.0008(12)$ & $0.0008(10)$ & $-0.0041(11)$ \\
C4AB & $0.0337(13)$ & $0.0346(14)$ & $0.0392(12)$ & $-0.0010(11)$ & $0.0011(10)$ & $0.0001(10)$ \\
C5B & $0.0418(15)$ & $0.0499(17)$ & $0.0367(12)$ & $0.0016(13)$ & $0.0065(10)$ & $0.0000(12)$ \\
C6B & $0.0420(16)$ & $0.0505(18)$ & $0.0546(15)$ & $0.0029(14)$ & $0.0138(12)$ & $-0.0033(14)$ \\
C7B & $0.0314(14)$ & $0.0487(18)$ & $0.0643(16)$ & $0.0060(13)$ & $0.0031(12)$ & $0.0073(15)$ \\
C8B & $0.0398(15)$ & $0.0471(17)$ & $0.0441(13)$ & $0.0003(13)$ & $-0.0042(11)$ & $0.0064(12)$ \\
C8AB & $0.0360(14)$ & $0.0402(15)$ & $0.0388(12)$ & $-0.0019(12)$ & $0.0028(10)$ & $-0.0012(11)$ \\
N9B & $0.0391(14)$ & $0.072(2)$ & $0.0366(11)$ & $0.0112(13)$ & $-0.0015(10)$ & $-0.0058(12)$ \\
& & & & & & \\
\hline & & & & & & \\
\hline
\end{tabular}

Geometric parameters $\left(A,{ }^{o}\right)$

\begin{tabular}{llll}
\hline $\mathrm{O} 1 \mathrm{~W}-\mathrm{H} 1 \mathrm{~W} 1$ & $0.91(2)$ & $\mathrm{N} 9 \mathrm{~A}-\mathrm{H} 9 \mathrm{AA}$ & $0.91(4)$ \\
$\mathrm{O} 1 \mathrm{~W}-\mathrm{H} 2 \mathrm{~W} 1$ & $0.89(2)$ & $\mathrm{N} 9 \mathrm{~A}-\mathrm{H} 9 \mathrm{AB}$ & $0.94(5)$ \\
$\mathrm{O} 2 \mathrm{~W}-\mathrm{H} 1 \mathrm{~W} 2$ & $0.91(2)$ & $\mathrm{N} 1 \mathrm{~B}-\mathrm{C} 2 \mathrm{~B}$ & $1.309(4)$ \\
$\mathrm{O} 2 \mathrm{~W}-\mathrm{H} 2 \mathrm{~W} 2$ & $0.89(2)$ & $\mathrm{N} 1 \mathrm{~B}-\mathrm{C} 8 \mathrm{AB}$ & $1.383(4)$ \\
$\mathrm{N} 1 \mathrm{~A}-\mathrm{C} 2 \mathrm{~A}$ & $1.315(4)$ & $\mathrm{C} 2 \mathrm{~B}-\mathrm{N} 3 \mathrm{~B}$ & $1.340(4)$ \\
$\mathrm{N} 1 \mathrm{~A}-\mathrm{C} 8 \mathrm{AA}$ & $1.393(4)$ & $\mathrm{C} 2 \mathrm{~B}-\mathrm{H} 2 \mathrm{BA}$ & 0.9300 \\
$\mathrm{~N} 1 \mathrm{~A}-\mathrm{H} 1 \mathrm{~A}$ & $1.03(4)$ & $\mathrm{N} 3 \mathrm{~B}-\mathrm{C} 4 \mathrm{~B}$ & $1.347(4)$ \\
$\mathrm{C} 2 \mathrm{~A}-\mathrm{N} 3 \mathrm{~A}$ & $1.328(4)$ & $\mathrm{C} 4 \mathrm{~B}-\mathrm{N} 9 \mathrm{~B}$ & $1.323(4)$ \\
$\mathrm{C} 2 \mathrm{~A}-\mathrm{H} 2 \mathrm{AA}$ & 0.9300 & $\mathrm{C} 4 \mathrm{~B}-\mathrm{C} 4 \mathrm{AB}$ & $1.443(4)$ \\
$\mathrm{N} 3 \mathrm{~A}-\mathrm{C} 4 \mathrm{~A}$ & $1.363(4)$ & $\mathrm{C} 4 \mathrm{AB}-\mathrm{C} 5 \mathrm{~B}$ & $1.408(4)$ \\
$\mathrm{C} 4 \mathrm{~A}-\mathrm{N} 9 \mathrm{~A}$ & $1.293(4)$ & $\mathrm{C} 4 \mathrm{AB}-\mathrm{C} 8 \mathrm{AB}$ & $1.409(4)$
\end{tabular}




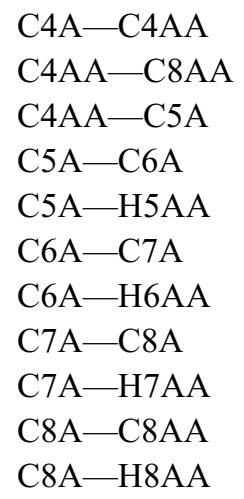

$1.455(4)$

$1.391(4)$

$1.397(4)$

$1.356(5)$

0.9300

$1.403(5)$

0.9300

$1.372(5)$

0.9300

$1.394(4)$

0.9300

$95(6)$

87 (6)

$120.3(2)$

119 (2)

$120(2)$

$125.0(3)$

117.5

117.5

$118.0(2)$

$116.2(3)$

$122.9(3)$

$120.8(2)$

$119.2(3)$

$116.8(2)$

$124.0(2)$

120.4 (3)

119.8

119.8

$120.0(3)$

120.0

120.0

120.9 (3)

119.5

119.5

$118.6(3)$

120.7

120.7

$119.0(3)$

120.8 (3)

$120.2(2)$

120 (2)

$-0.2(5)$

$0.2(5)$

$179.0(3)$

$-0.3(4)$
$\mathrm{C} 5 \mathrm{~B}-\mathrm{C} 6 \mathrm{~B}$

$\mathrm{C} 5 \mathrm{~B}-\mathrm{H} 5 \mathrm{BA}$

$\mathrm{C} 6 \mathrm{~B}-\mathrm{C} 7 \mathrm{~B}$

$\mathrm{C} 6 \mathrm{~B}-\mathrm{H} 6 \mathrm{BA}$

$\mathrm{C} 7 \mathrm{~B}-\mathrm{C} 8 \mathrm{~B}$

C7B-H7BA

$\mathrm{C} 8 \mathrm{~B}-\mathrm{C} 8 \mathrm{AB}$

$\mathrm{C} 8 \mathrm{~B}-\mathrm{H} 8 \mathrm{BA}$

N9B-H9BA

$\mathrm{N} 9 \mathrm{~B}-\mathrm{H} 9 \mathrm{BB}$

$\mathrm{C} 4 \mathrm{~A}-\mathrm{N} 9 \mathrm{~A}-\mathrm{H} 9 \mathrm{AB}$

H9AA-N9A-H9AB

$\mathrm{C} 2 \mathrm{~B}-\mathrm{N} 1 \mathrm{~B}-\mathrm{C} 8 \mathrm{AB}$

$\mathrm{N} 1 \mathrm{~B}-\mathrm{C} 2 \mathrm{~B}-\mathrm{N} 3 \mathrm{~B}$

$\mathrm{N} 1 \mathrm{~B}-\mathrm{C} 2 \mathrm{~B}-\mathrm{H} 2 \mathrm{BA}$

$\mathrm{N} 3 \mathrm{~B}-\mathrm{C} 2 \mathrm{~B}-\mathrm{H} 2 \mathrm{BA}$

$\mathrm{C} 2 \mathrm{~B}-\mathrm{N} 3 \mathrm{~B}-\mathrm{C} 4 \mathrm{~B}$

$\mathrm{N} 9 \mathrm{~B}-\mathrm{C} 4 \mathrm{~B}-\mathrm{N} 3 \mathrm{~B}$

$\mathrm{N} 9 \mathrm{~B}-\mathrm{C} 4 \mathrm{~B}-\mathrm{C} 4 \mathrm{AB}$

$\mathrm{N} 3 \mathrm{~B}-\mathrm{C} 4 \mathrm{~B}-\mathrm{C} 4 \mathrm{AB}$

$\mathrm{C} 5 \mathrm{~B}-\mathrm{C} 4 \mathrm{AB}-\mathrm{C} 8 \mathrm{AB}$

$\mathrm{C} 5 \mathrm{~B}-\mathrm{C} 4 \mathrm{AB}-\mathrm{C} 4 \mathrm{~B}$

$\mathrm{C} 8 \mathrm{AB}-\mathrm{C} 4 \mathrm{AB}-\mathrm{C} 4 \mathrm{~B}$

$\mathrm{C} 6 \mathrm{~B}-\mathrm{C} 5 \mathrm{~B}-\mathrm{C} 4 \mathrm{AB}$

$\mathrm{C} 6 \mathrm{~B}-\mathrm{C} 5 \mathrm{~B}-\mathrm{H} 5 \mathrm{BA}$

$\mathrm{C} 4 \mathrm{AB}-\mathrm{C} 5 \mathrm{~B}-\mathrm{H} 5 \mathrm{BA}$

$\mathrm{C} 5 \mathrm{~B}-\mathrm{C} 6 \mathrm{~B}-\mathrm{C} 7 \mathrm{~B}$

$\mathrm{C} 5 \mathrm{~B}-\mathrm{C} 6 \mathrm{~B}-\mathrm{H} 6 \mathrm{BA}$

$\mathrm{C} 7 \mathrm{~B}-\mathrm{C} 6 \mathrm{~B}-\mathrm{H} 6 \mathrm{BA}$

$\mathrm{C} 8 \mathrm{~B}-\mathrm{C} 7 \mathrm{~B}-\mathrm{C} 6 \mathrm{~B}$

$\mathrm{C} 8 \mathrm{~B}-\mathrm{C} 7 \mathrm{~B}-\mathrm{H} 7 \mathrm{BA}$

$\mathrm{C} 6 \mathrm{~B}-\mathrm{C} 7 \mathrm{~B}-\mathrm{H} 7 \mathrm{BA}$

$\mathrm{C} 7 \mathrm{~B}-\mathrm{C} 8 \mathrm{~B}-\mathrm{C} 8 \mathrm{AB}$

$\mathrm{C} 7 \mathrm{~B}-\mathrm{C} 8 \mathrm{~B}-\mathrm{H} 8 \mathrm{BA}$

$\mathrm{C} 8 \mathrm{AB}-\mathrm{C} 8 \mathrm{~B}-\mathrm{H} 8 \mathrm{BA}$

$\mathrm{N} 1 \mathrm{~B}-\mathrm{C} 8 \mathrm{AB}-\mathrm{C} 8 \mathrm{~B}$

$\mathrm{N} 1 \mathrm{~B}-\mathrm{C} 8 \mathrm{AB}-\mathrm{C} 4 \mathrm{AB}$

$\mathrm{C} 8 \mathrm{~B}-\mathrm{C} 8 \mathrm{AB}-\mathrm{C} 4 \mathrm{AB}$

$\mathrm{C} 4 \mathrm{~B}-\mathrm{N} 9 \mathrm{~B}-\mathrm{H} 9 \mathrm{BA}$

$\mathrm{C} 4 \mathrm{~B}-\mathrm{N} 9 \mathrm{~B}-\mathrm{H} 9 \mathrm{BB}$

H9BA-N9B-H9BB

$\mathrm{C} 8 \mathrm{AB}-\mathrm{N} 1 \mathrm{~B}-\mathrm{C} 2 \mathrm{~B}-\mathrm{N} 3 \mathrm{~B}$

$\mathrm{N} 1 \mathrm{~B}-\mathrm{C} 2 \mathrm{~B}-\mathrm{N} 3 \mathrm{~B}-\mathrm{C} 4 \mathrm{~B}$

$\mathrm{C} 2 \mathrm{~B}-\mathrm{N} 3 \mathrm{~B}-\mathrm{C} 4 \mathrm{~B}-\mathrm{N} 9 \mathrm{~B}$

$\mathrm{C} 2 \mathrm{~B}-\mathrm{N} 3 \mathrm{~B}-\mathrm{C} 4 \mathrm{~B}-\mathrm{C} 4 \mathrm{AB}$
1.364 (4)

0.9300

1.399 (4)

0.9300

1.371 (4)

0.9300

$1.402(4)$

0.9300

$0.95(4)$

$0.80(4)$

$120(3)$

120 (4)

$116.4(2)$

$128.1(3)$

115.9

115.9

$117.4(2)$

$117.4(3)$

$122.3(3)$

$120.3(2)$

$119.2(3)$

$124.1(2)$

$116.7(2)$

$120.6(2)$

119.7

119.7

$120.1(3)$

120.0

120.0

$120.6(3)$

119.7

119.7

$120.3(2)$

119.9

119.9

119.7 (2)

121.1 (3)

119.2 (3)

119 (2)

120 (3)

$120(3)$

$-0.2(5)$

$-1.5(5)$

-179.1 (3)

$1.6(5)$ 
